\title{
Norepinephrine metabolite DOPEGAL activates AEP and pathological Tau aggregation in locus coeruleus
}

\author{
Seong Su Kang, ${ }^{1}$ Xia Liu, ${ }^{1}$ Eun Hee Ahn, ${ }^{1}$ Jie Xiang, ${ }^{1}$ Fredric P. Manfredsson, ${ }^{2}$ Xifei Yang, ${ }^{3}$ Hongbo R. Luo, ${ }^{4}$ L. Cameron Liles, ${ }^{5}$ \\ David Weinshenker, ${ }^{5}$ and Keqiang $\mathrm{Ye}^{1}$ \\ 'Department of Pathology and Laboratory Medicine, Emory University School of Medicine, Atlanta, Georgia, USA. ${ }^{2}$ Translational Science and Molecular Medicine, Michigan State University, College of \\ Human Medicine, Grand Rapids, Michigan, USA. ${ }^{3}$ Key Laboratory of Modern Toxicology of Shenzhen, Shenzhen Center for Disease Control and Prevention, Shenzhen, China. ${ }^{4}$ Department of Pathology and \\ Laboratory Medicine, Harvard Medical School and Children's Hospital, Boston, Massachusetts, USA. ${ }^{5}$ Department of Human Genetics, Emory University School of Medicine, Atlanta, Georgia, USA.
}

\begin{abstract}
Aberrant Tau inclusions in the locus coeruleus (LC) are the earliest detectable Alzheimer's disease-like (AD-like) neuropathology in the human brain. However, why LC neurons are selectively vulnerable to developing early Tau pathology and degenerating later in disease and whether the LC might seed the stereotypical spread of Tau pathology to the rest of the brain remain unclear. Here, we show that 3,4-dihydroxyphenylglycolaldehyde, which is produced exclusively in noradrenergic neurons by monoamine oxidase A metabolism of norepinephrine, activated asparagine endopeptidase that cleaved Tau at residue N368 into aggregation- and propagation-prone forms, thus leading to LC degeneration and the spread of Tau pathology. Activation of asparagine endopeptidase-cleaved Tau aggregation in vitro and in intact cells was triggered by 3,4-dihydroxyphenylglycolaldehyde, resulting in LC neurotoxicity and propagation of pathology to the forebrain. Thus, our findings reveal that norepinephrine metabolism and Tau cleavage represent the specific molecular mechanism underlying the selective vulnerability of LC neurons in AD.
\end{abstract}

\section{Introduction}

Alzheimer's disease (AD) is an insidious, debilitating illness characterized by amyloid- $\beta$ (A- $\beta$ ) plaques and Tau neurofibrillary tangles (NFTs), as well as neuronal cell death and neuroinflammation. Despite intense research into the pathologic hallmarks of $\mathrm{AD}$, no disease-modifying therapies have emerged (1), and recent research efforts are focused on early AD detection and treatment. One area of interest in early AD involves the locus coeruleus (LC), the major brainstem noradrenergic nucleus that innervates and supplies norepinephrine (NE) to the forebrain and regulates attention, arousal, cognition, and stress responses (2). Hyperphosphorylated Tau, a "pretangle" form of the protein prone to aggregation, appears in the LC before any other area of the brain and is now considered the earliest detectable AD-like neuropathology (3-7). Moreover, degeneration of the LC is a ubiquitous feature of midto late-stage disease, contributing to the progression of forebrain pathology and cognitive impairment $(6,8-12)$, whereas restoration of noradrenergic signaling can ameliorate AD-like symptoms in animal models $(13,14)$. The progression of AD pathology follows a remarkably systematic pattern across individuals, and aberrant forms of Tau are capable of neuron-to-neuron propagation via a prion-like process of corruptive templating (15). Hyperphosphorylated Tau appears exclusively in the LC in Braak stage 0 , spreads to the entorhinal cortex $(\mathrm{Cx})$ in Braak stages I and II, then propagates

Conflict of interest: The authors have declared that no conflict of interest exists. Copyright: (C) 2020, American Society for Clinical Investigation.

Submitted: May 21, 2019; Accepted: October 3, 2019; Published: December 3, 2019.

Reference information: / Clin Invest. 2020;130(1):422-437.

https://doi.org/10.1172/JCI130513. to the hippocampus and frontal $\mathrm{Cx}$ in stages III-VI (3). Because hyperphosphorylated Tau can first be detected in the LC, and the LC sends dense projections to other vulnerable brain regions that display early Tau pathology (e.g., the transentorhinal $\mathrm{Cx}$ and hippocampus), some suggest that the LC might be one of the origins of Tau neuropathology in $\mathrm{AD}(4,5)$. Although several theories have emerged to explain the selective vulnerability of LC neurons in $\mathrm{AD}$, such as their unique anatomical, electrophysiological, morphological, and neurochemical characteristics $(7,16)$, the underlying molecular mechanisms have yet to be identified.

Asparagine endopeptidase (AEP; gene name $L G M N$ ) is a lysosomal endopeptidase that specifically cleaves its substrates after asparagine under conditions of acidosis (17). Recently, we reported that AEP acts as a delta-secretase that cleaves both amyloid precursor protein (APP) and Tau in an age-dependent manner in mouse and human AD brains $(18,19)$. AEP cuts APP at the N373 and $\mathrm{N} 585$ residues in the ectodomain and facilitates $A-\beta$ production by decreasing the steric hindrance for $\beta$-secretase. Depletion of AEP significantly reduces A- $\beta$ production and senile plaque formation in 5XFAD transgenic mice, leading to the restoration of synaptic activity and cognitive functions (19). In addition, AEP cleaves Tau at N255 and N368 and abolishes its microtubule assembly activity, resulting in hyperphosphorylation, aggregation, and NFT formation (18). Of note, the AEP-generated Tau 1-368 fragment is highly neurotoxic. Genetic deletion or pharmacological inhibition of AEP in transgenic mice that overexpress mutant human APP or aggregation-prone mutant human Tau prevents APP N585 and Tau N368 cleavage, ameliorates A- $\beta$ and Tau pathology, and reverses synaptic plasticity and cognitive deficits $(18,20)$. Thus, AEP plays a critical role in regulating A- $\beta$ and Tau pathogenesis. Most recently, 
we reported that AEP is activated by 3,4-dihydroxyphenylacetaldehyde (DOPAL), a highly toxic and oxidative dopamine (DA) metabolite, in the substantia nigra (21). Active AEP subsequently cleaves $\alpha$-synuclein at N103 and promotes its aggregation and dopaminergic neuronal loss, leading to motor dysfunction in animal models of Parkinson's disease (PD) (22).

Analogous to toxic DA metabolites killing substantia nigra pars compacta neurons in patients with $\mathrm{PD}$, the noradrenergic phenotype of LC neurons itself may contribute to the vulnerability of these cells in AD. NE is converted into 3,4-dihydroxyphenylglycolaldehyde (DOPEGAL) by monoamine oxidase A (MAO-A) during the normal life cycle of catecholamine production and transmission, but is increased in the degenerating LC neurons of patients with AD (23-25). Injection of DOPEGAL into rodent brains elicits adrenergic neuronal loss (26), and DOPEGAL toxicity is likely due to the generation of free radicals and activation of mitochondrial permeability transition (27). The activation of AEP by DOPAL suggested to us that it might also be activated by DOPEGAL, thus triggering a cascade of events leading to Tau cleavage, hyperphosphorylation, aggregation, and neurotoxicity in LC neurons.

\section{Results}

DOPEGAL triggers Tau aggregation in vitro and in cells. To examine whether DOPEGAL directly modifies Tau, we incubated recombinant WT Tau with DOPEGAL for 24 hours. We found DOPEGAL induced Tau aggregation, indicated by the accumulation of high-molecular-weight bands, in a concentrationdependent manner. As expected, AEP-truncated Tau N368 recombinant proteins were more prone to aggregation than fulllength Tau (Figure 1A). To explore the specificity of DOPEGAL, we tested various catecholamines and their oxidative metabolites $(500 \mu \mathrm{M})$. Compared with vehicle control, DOPEGAL strongly provoked Tau aggregation, while NE, DA, and DOPAL instead caused Tau protein degradation (Figure 1B). Time course and dose-response assays demonstrated that both DA and NE triggered dose- and time-dependent degradation of Tau, whereas DOPAL elicited Tau aggregation at lower doses $(2.5-25 \mu \mathrm{M})$ but promoted degradation at higher doses (Supplemental Figure 1, $\mathrm{A}-\mathrm{C}$; supplemental material available online with this article; https://doi.org/10.1172/JCI130513DS1). To investigate whether DOPEGAL elicits Tau fibrillization in vitro, we monitored the fluorescent intensity of recombinant Tau in the presence of Thiofla$\operatorname{vin} \mathrm{T}$ (ThioT) $(30 \mu \mathrm{M})$. Compared with vehicle control, DOPEGAL strongly augmented Tau fibrillization over time, as revealed by the high-intensity ThioT fluorescence of Tau preformed fibrils (PFFs), while NE and DOPAL inhibited Tau fibrillization (Figure 1C). Transmission electronic microscopy analysis confirmed that Tau PFFs formed tight fibrillar structures following DOPEGAL exposure (Supplemental Figure 1D).

To assess whether DOPEGAL provokes Tau aggregation in intact cells, we transfected noradrenergic-like SH-SY5Y cells with WT human Tau, followed by treatment with different catecholamines or their oxidative metabolites for 24 hours. Immunoblotting revealed that DOPEGAL (but not DA or NE) produced demonstrable Tau aggregation, which was in alignment with its hyperphosphorylation (AT8-positive) status (Figure 1D, top 2 panels). The lack of an effect for NE is consistent with the lim- ited conversion capacity for exogenous NE in SH-SY5Y cells due to the low expression of MAO-A. We next determined whether DOPEGAL, like DOPAL, activates AEP. We found DOPEGAL upregulated total AEP levels and its proteolytic activation, as well as the abundance of the AEP cleavage product Tau N368 (Figure 1D, bottom 2 panels, Figure 1E) and induced SH-SY5Y cell death (Figure 1, F-H). DOPAL was less effective in these measures, while NE and DA failed to activate AEP or cause toxicity. To ascertain whether Tau is required for DOPEGAL-elicited cell death, we prepared primary cortical neurons from neonatal WT and Tau knockout $\left(\mathrm{Tau}^{-/}\right)$mice and treated them with DOPEGAL. As expected, DOPEGAL triggered demonstrable Tau hyperphosphorylation, aggregation, and N368 cleavage in WT, but not Tau ${ }^{-/}$, neurons (Figure 1I). Importantly, although AEP was similarly activated by DOPEGAL in both WT and $\mathrm{Tau}^{-/-}$neurons (Figure 1J), its toxicity was significantly attenuated in $\mathrm{Tau}^{-/-}$neurons (Figure 1, $\mathrm{K}-\mathrm{M})$, suggesting that Tau is necessary for the full expression of DOPEGAL-induced cell death.

NE oxidation by MAO-A facilitates AEP-cleaved Tau N368 cytotoxicity. Oxidative deamination of NE by mitochondrial MAO-A generates DOPEGAL and $\mathrm{H}_{2} \mathrm{O}_{2}$, leading to oxidative stress (28, 29). To test whether MAO-mediated metabolism of NE results in oxidative stress and AEP activation, we transfected SH-SY5Y cells (30) with MAO-A or MAO-B. We found that MAO overexpression increased DOPEGAL levels, AEP enzymatic activity, and Tau N368 cleavage (Supplemental Figure 2, A-D), which was mimicked by $\mathrm{H}_{2} \mathrm{O}_{2}$ and prevented by the MAO-A inhibitor clorgyline, indicating the importance of oxidative stress (Supplemental Figure 2, E-G). Interestingly, $\mathrm{H}_{2} \mathrm{O}_{2}$ also increased MAO-A expression, which was blunted by clorgyline. To evaluate the contribution of NE metabolism, we transfected SH-SY5Y cells with siRNA against dopamine $\beta$-hydroxylase (DBH), which is required for NE synthesis (31). DBH depletion reduced AEP activity, diminished Tau N368 cleavage, and partially ameliorated the deleterious effects of $\mathrm{H}_{2} \mathrm{O}_{2}$ (Supplemental Figure 2, $\mathrm{H}$ and I). Combined, these results suggest that oxidative metabolism of NE to DOPEGAL by MAO activates AEP and Tau N368 cleavage in catecholaminergic $\mathrm{SH}-\mathrm{SY} 5 \mathrm{Y}$ cells.

To explore the neurotoxicity of AEP-cleaved Tau, we infected SH-SY5Y cells with AAV encoding full-length WT Tau, P301S Tau, the N368 Tau fragment, or uncleavable P301S/N255/368A Tau, and determined cell death using TUNEL staining and lactate dehydrogenase (LDH) assay (Supplemental Figure 2, J-M). WT Tau promoted cell death, which was escalated by the AEP-cleaved Tau N368 fragment. Notably, N368 Tau displayed neurotoxicity comparable with Tau P301S, whereas prevention of AEP cleavage blunted Tau P301S toxicity. These results indicate that AEP cleavage contributes to Tau neurotoxicity.

To ascertain whether DOPEGAL regulates Tau neurotoxicity, we overexpressed MAO-A or MAO-B in SH-SY5Y cells in combination with AAV-Tau. TUNEL and $\mathrm{LDH}$ analysis revealed that both MAO-A and MAO-B overexpression significantly activated AEP, increased Tau N368 cleavage, and magnified Tau neurotoxicity as measured by TUNEL, LDH, and loss of the catecholaminergic marker tyrosine hydroxylase (TH) (Supplemental Figure 3). By contrast, preventing DOPEGAL production by siRNA knockdown of DBH attenuated AEP activation and Tau-triggered cell 
A

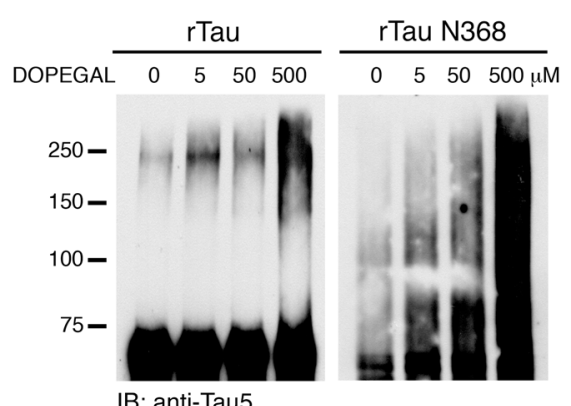

B
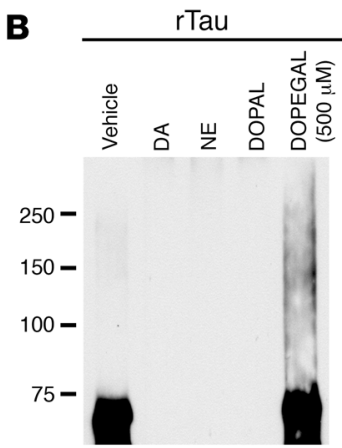

IB: anti-Tau5

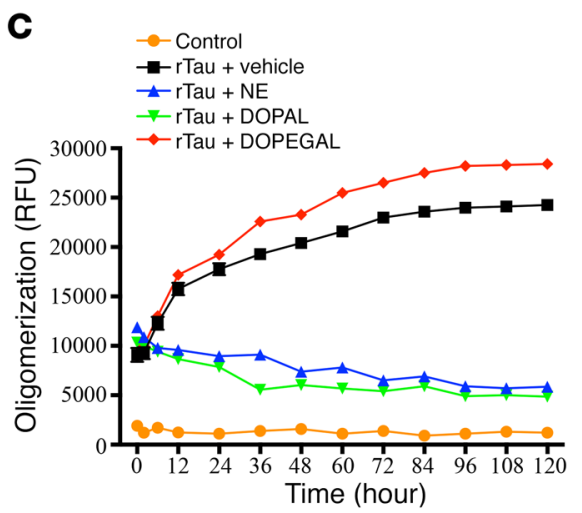

D

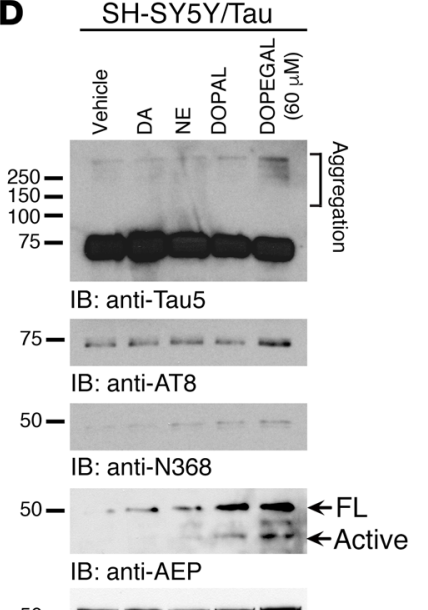

E
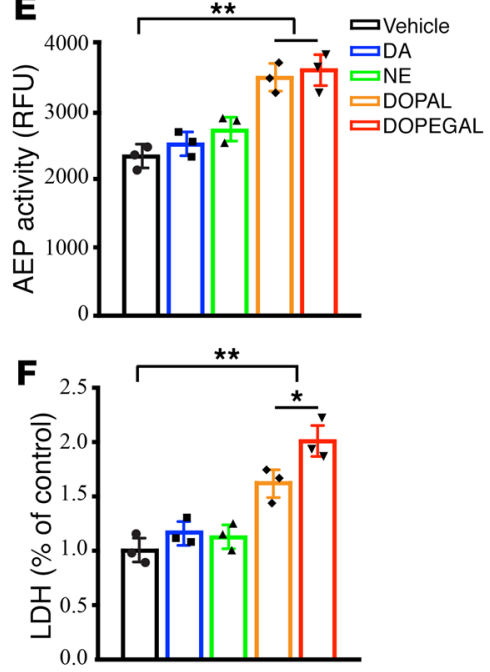

G

SH-SY5YIAAV-Tau
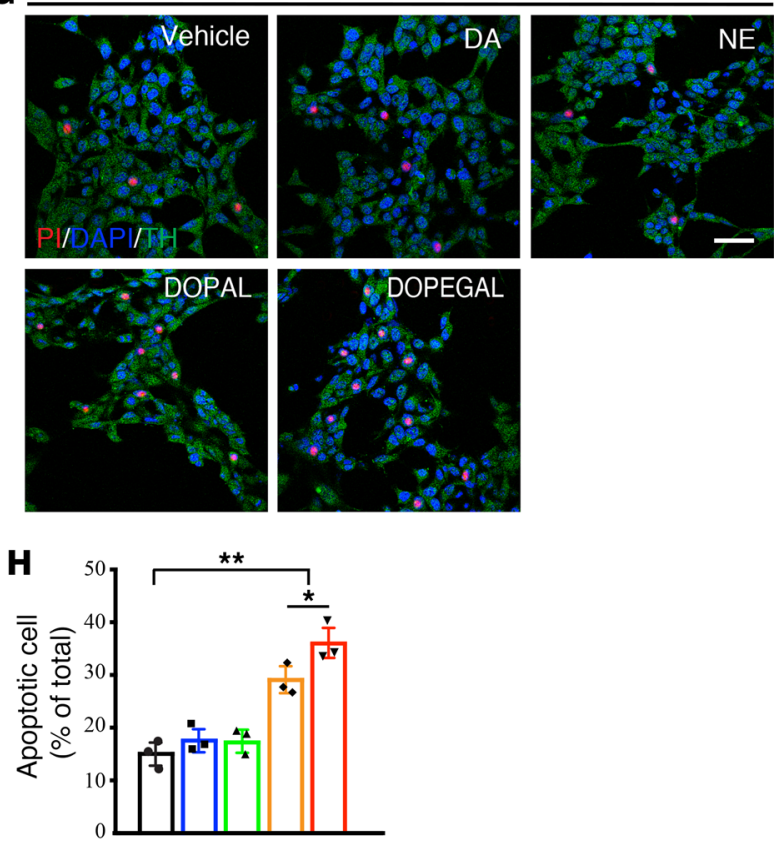

$\mathbf{L}$

Cortical neuron
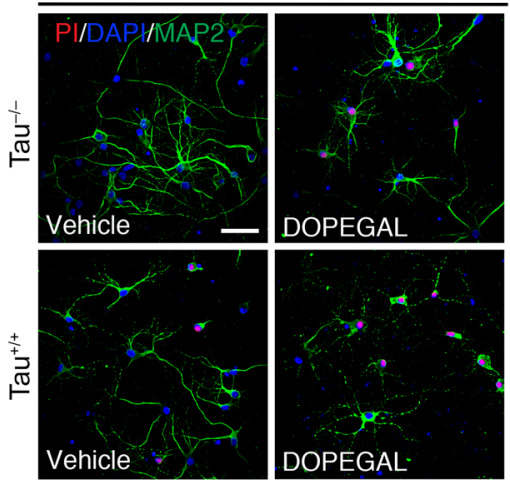

M

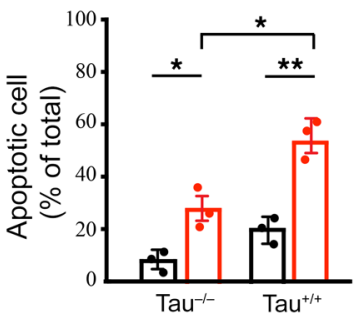

Figure 1. The NE metabolite DOPEGAL triggers Tau aggregation in vitro. (A) Recombinant Tau ( $r T a u)$ or rTau N368 (1 $\mu$ g) was incubated with DOPEGAL (0, 5 , 50, or $500 \mu \mathrm{M}$ ) for 24 hours, and aggregation was detected by Western blot using Tau5 antibody. (B) rTau (1 $\mu \mathrm{g})$ was incubated with vehicle, DA, NE, DOPAL, or DOPECAL $(500 \mu \mathrm{M})$ for 24 hours, and aggregation was detected by Western blot using Tau 5 antibody. (C) rTau (1 $\mu \mathrm{g})$ was incubated with vehicle or DOPECAL, and ThioT assay was used to evaluate the kinetics of aggregation of Tau over 120 hours. SH-SY5Y cells were infected with hTau virus, then exposed to DA, NE, DOPAL, or DOPEGAL $(60 \mu \mathrm{M})$ for 24 hours. (D) Western blot analysis was conducted with cell lysates using antibodies against AEP and different forms of Tau. (E) AEP activity was analyzed by the enzymatic assay. Data are shown as mean \pm SEM ( $n=3$ per group). ${ }^{*} P<0.05$ and ${ }^{* *} P<0.01$, by 1 -way ANOVA. Cell death was analyzed by LDH assay (F) and propidium iodide staining (G-H). Scale bar: $50 \mu \mathrm{m}$. Data are shown as the mean \pm SEM ( $n=3$ per group). . $P<0.05$ and ${ }^{* *} P<0.01$, by 1-way ANOVA. Primary cortical neurons were cultured from Tau ${ }^{+/}$and Tau ${ }^{-1-}$ mice and exposed to vehicle or DOPECAL (60 $\mu$ M) for 24 hours. (I) Western blot analysis was conducted with cell lysates using antibodies against AEP and different forms of Tau. (J) AEP activity was analyzed by the enzymatic assay. Data are shown as the mean \pm SEM ( $n=3$ per group). ${ }^{*} P<0.05$ and ${ }^{*} P<0.01$, by 2 -tailed $t$ test. Cell death was analyzed by LDH assay (K) and propidium iodide staining ( $\mathbf{L}$ and $\mathbf{M})$. Scale bar: $50 \mu \mathrm{m}$. Data are shown as the mean \pm SEM ( $n=3$ per group). ${ }^{*} P<0.05$ and ${ }^{* *} P<0.01$, by 2 -way ANOVA. 
A
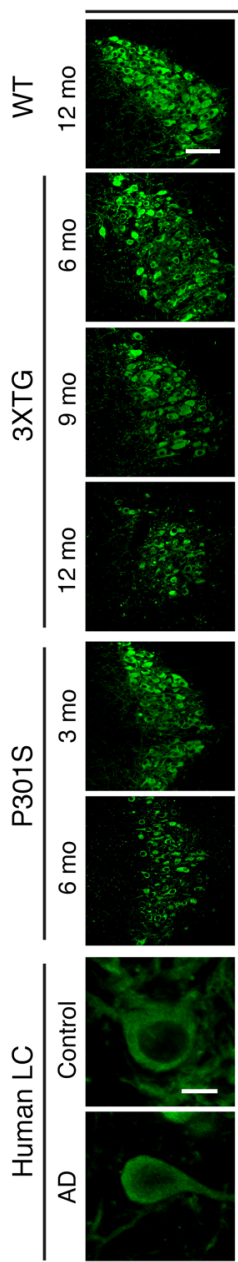
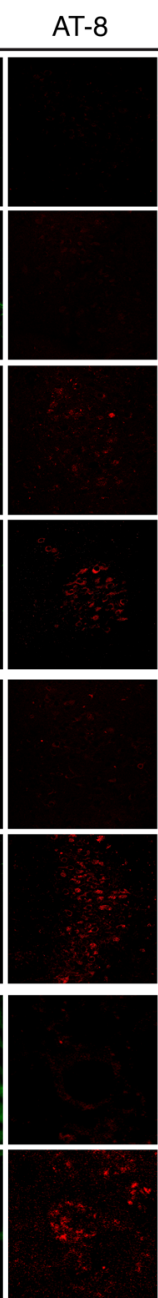

Merge
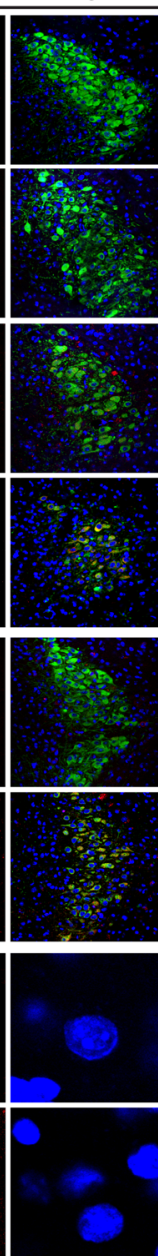

$60 x$
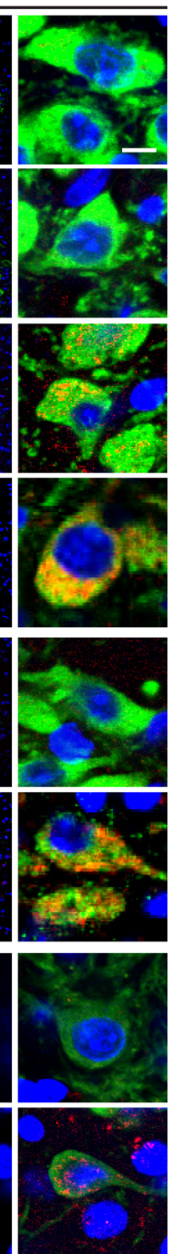

$\mathrm{TH}$
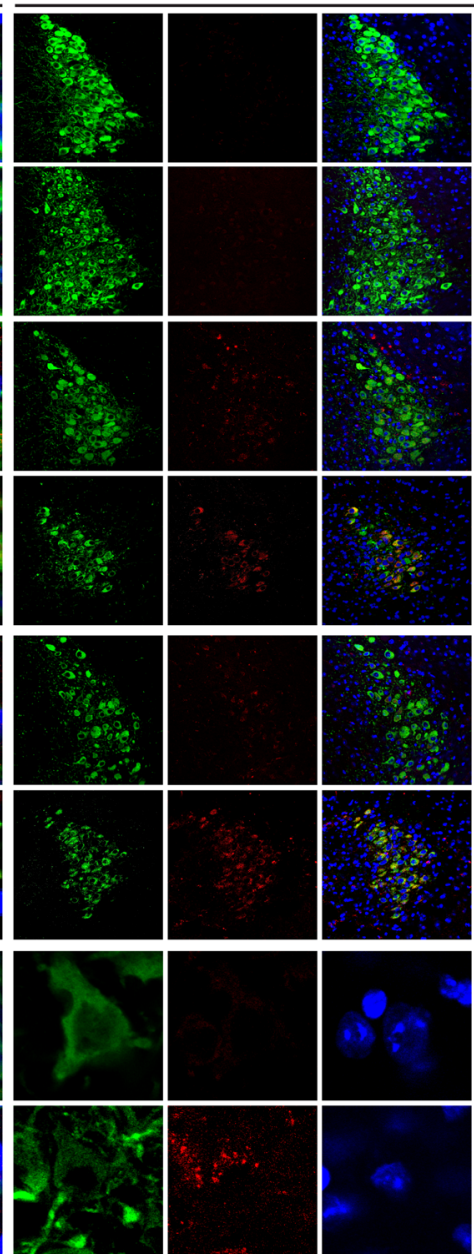

TauN368
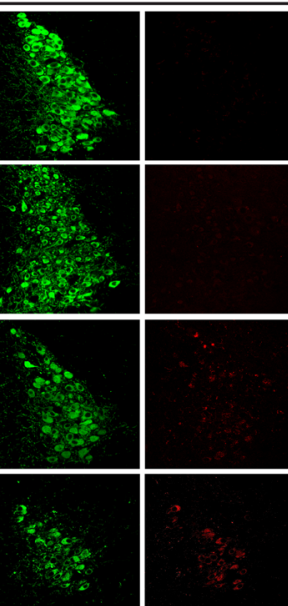

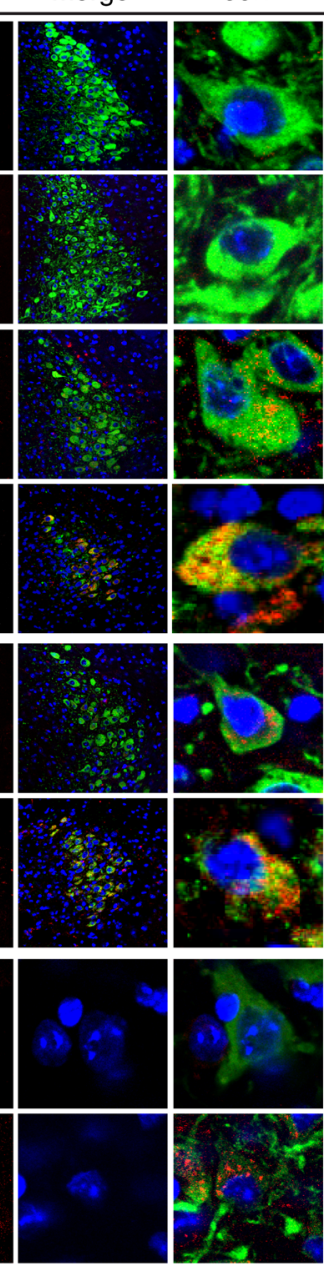

$60 x$
B
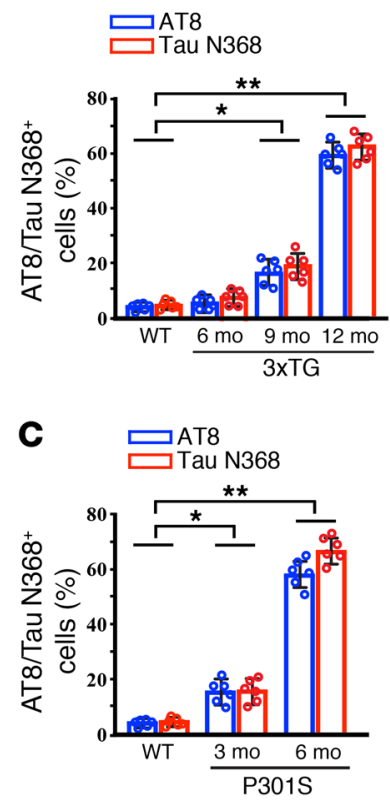

D $\quad$ ॠTA8 N368

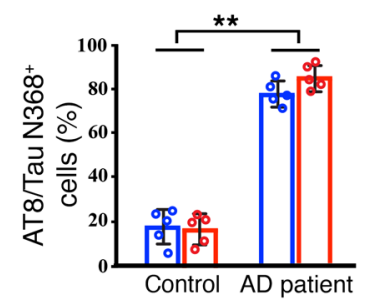

Figure 2. Tau is cleaved at N368 and hyperphosphorylated in the LC region of transgenic AD mouse models and human postmortem AD brains. WT, 3xTg, and P3015 mouse brains from various ages, as well as postmortem human control and AD brains, were examined for Tau pathology in the LC by immunohistochemistry. (A) Representative images of staining for TH (green), AT8 or Tau N368 (red), and DAPI (blue). Scale bar: $100 \mu \mathrm{m}$ ( $\times 60$ panel is 20 $\mu \mathrm{m})$. (B-D) Quantification of AT8 and Tau N368 cells in 3XTC (B), Tau P301S mice (C), and human brains of controls and patients with AD (D). Data are shown as mean \pm SEM ( $n=5-6$ per group). ${ }^{*} P<0.05$ and ${ }^{* *} P<0.01$, by 2 -way ANOVA.

death. These findings reveal that a proportion of Tau neurotoxicity is NE-dependent.

Our data support a model whereby MAO-A oxidizes NE into DOPEGAL and elicits oxidative stress, triggering AEP activation and Tau N368 cleavage and cytotoxicity. To further explore this idea, we overexpressed MAO-A and MAO-B into SH-SY5Y cells and interrogated their roles in AEP activation in the presence of specific inhibitors. We confirmed that overexpression of either MAO-A or MAO-B substantially provoked AEP activation, and blockade of these enzymes by small molecular inhibitors attenuated AEP enzymatic activity, Tau hyperphosphorylation, and N368 cleavage (Supplemental Figure 4, A-E). To test whether elevation of NE in SH-SY5Y cells facilitates MAO-A cytotoxicity, we transfected cells with MAO-A, followed by treatment with ascorbic acid, which stimulates NE biosynthesis (32), the DA and NE precursor L-3,4-dihydroxyphenylalanine (L-DOPA), or the synthetic NE precursor L-3,4-dihydroxyphenylserine (L-DOPS). Although these compounds alone barely activated AEP, both L-DOPS and ascorbic acid significantly increased AEP activation, Tau N368 cleavage, and AT8 immunoreactivity in the presence of elevated MAO-A (Supplemental Figure 4, F and G). Interestingly, both L-DOPA and L-DOPS, but not ascorbic acid, exhibited cytotoxicity on their own, which was facilitated by MAO-A overexpression (Supplemental Figure 4H). The limited cytotoxicity of ascorbic acid may be attributed to its antioxidant activity.

DOPEGAL is metabolized in vivo via reduction by aldehyde reductase (AR) and further oxidation by aldehyde dehydrogenase (ALDH). To determine whether accumulation of DOPEGAL via blockade of its metabolism augments its cytotoxicity, we treated SH-SY5Y cells with the AR inhibitor imirestat, the ALDH inhibitor daidzein, or both drugs in the presence or absence of MAO-A overexpression. Although no treatment was effective on its own, imirestat and the mixture of both inhibitors augmented AEP activation, Tau N368 cleavage, and AT8 abundance in combination with MAO-A (Supplemental Figure 4, I and J). LDH assay indicated that the inhibitor cocktail modestly stimulated cell death without MAO-A overexpression, while either imirestat or imirestat plus daidzein escalated MAO-A cytotoxicity (Supplemental Figure 

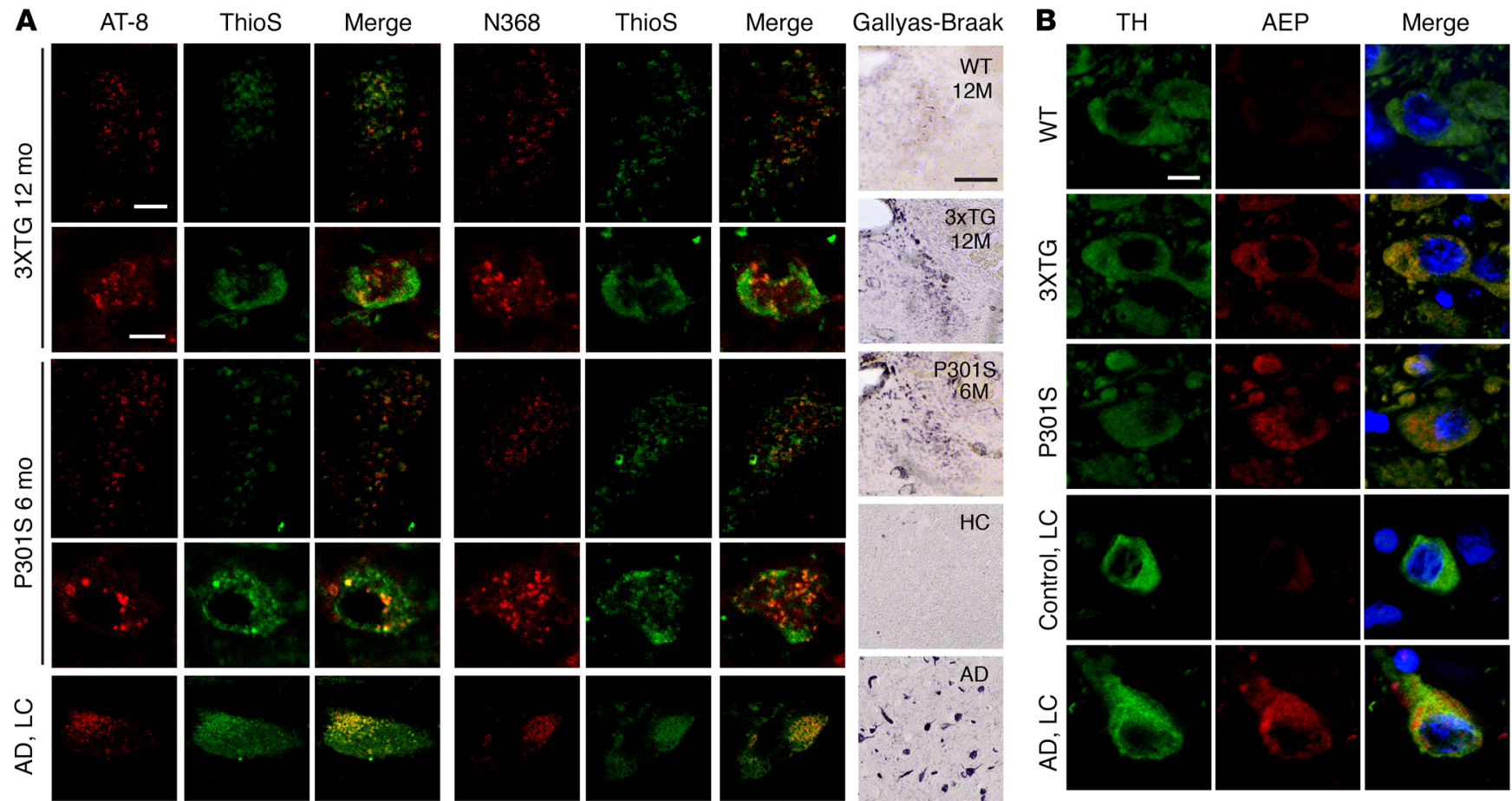

Figure 3. AEP and Tau fibrilization are activated in the LC region of transgenic AD mouse models and human postmortem AD brains. WT, 3XTg, and P3015 mouse, as well as postmortem human control and AD brains, were examined for Tau fibrilization in the LC by Gallyas-Braak staining. (A) Representative images of staining for AT8 or Tau N368 (red) and ThioS (green), and Gallyas-Braak. Scale bars: $100 \mu \mathrm{m}$ (top panel) and $20 \mu \mathrm{m}$ (second panel).

(B) Representative images of staining for TH (green), AEP (red), and DAPI (blue). Scale bar: $20 \mu \mathrm{m}$. $n=5-6$ per group.

$4 \mathrm{~K})$. These results suggest that AR is the major enzyme responsible for DOPEGAL metabolism in SH-SY5Y cells and that preventing DOPEGAL metabolism is deleterious.

Tau hyperphosphorylation and N368 cleavage are increased in the $L C$ of human AD subjects and transgenic AD mouse models. Hyperphosphorylated Tau in the LC is among the first detectable AD pathologies in postmortem human brains, appearing before Tau pathology in the EC and often decades prior to cognitive symptoms (5). To explore whether Tau N368 cleavage by AEP correlates with Tau hyperphosphorylation, we conducted immunofluorescence (IF) staining in the LC of postmortem human AD brains and different ages of transgenic AD mouse models. TH was used as a marker for noradrenergic LC neurons. AT8 and Tau N368 immunoreactivity were absent from WT mouse brains but appeared and accumulated together in a time-dependent manner in the LC of both 3xTg mice and Tau P301S mice. Compared with healthy controls, LC sections from AD subjects exhibited robust AT8 and Tau N368 signals (Figure 2, A-D). These results indicate that AEP is temporally activated in the LC, cleaving Tau N368 and triggering its hyperphosphorylation. To investigate whether the hyperphosphorylated Tau was aggregated, we performed IF costaining with AT8 or Tau N368 and Thioflavin S (ThioS). AT8/N368-positive Tau was also ThioS-positive in the LC region of 12-month-old 3xTg mice, 6-month-old Tau P301S mice, and human AD subjects (Figure $3 \mathrm{~A})$. We further confirmed Tau fibrilization by Gallyas-Braak staining (Figure $3 \mathrm{~A}$, right panels). IF costaining also showed that AEP fluorescence intensity was elevated in the LC of both $3 \mathrm{xTg}$ and Tau P301S mice compared with WT controls, similar to human
$\mathrm{AD}$ brains (Figure 3B). These findings demonstrate that activated AEP is abundant in the diseased LC, where it is associated with Tau N368, hyperphosphorylated Tau, and aggregation.

$N E$ is required for AEP activation and Tau N368 cleavage in the LC of Tau P301S mice. To investigate whether DOPEGAL is implicated in Tau pathology and LC neuronal degeneration, we crossed Tau P301S transgenic mice with DBH knockout $\left(\mathrm{DBH}^{-/}\right)$mice that lack NE completely (and thus cannot produce DOPEGAL) and found Tau hyperphosphorylation and N368 cleavage at different ages. $\mathrm{DBH}^{+/-}$littermates with normal NE levels were used as controls (33). At 3 months, AT8 and Tau N368 immunoreactivity in the LC was minimal in both Tau P301S/ $\mathrm{DBH}^{+/-}$and Tau P301S/ $\mathrm{DBH}^{-/-}$mice. Although the abundance of pathological Tau escalated in both genotypes at 6 to 9 months, AT8, Tau N368, and Gallyas-Braak silver staining were significantly reduced in Tau $\mathrm{P} 301 \mathrm{~S} / \mathrm{DBH}^{-/-}$mice (Figure 4, A-C). Consistent with our finding that suppression of NE production protected against Tau toxicity in vitro (Supplemental Figure 3), TH staining revealed a loss of LC neurons in Tau P301S/ $\mathrm{DBH}^{+/-}$mice at 9 months that was partially abrogated by $\mathrm{DBH}^{-/}$(Figure 5 , A and B). To explore whether manipulation of NE synthesis affects cognitive impairment in Tau P301S mice, we tested spatial learning and memory using the Morris water maze (MWM). Comparable age-dependent deficits were observed at 3 and 6 months in NE-deficient and NE-competent Tau P301S mice. However, at 9 months, the performance of Tau P301S/ $\mathrm{DBH}^{-/-}$mice was significantly better than Tau P301S/ $\mathrm{DBH}^{+/-}$mice on several measures, including distance to platform during training and percentage of time spent in the target quadrant 
A

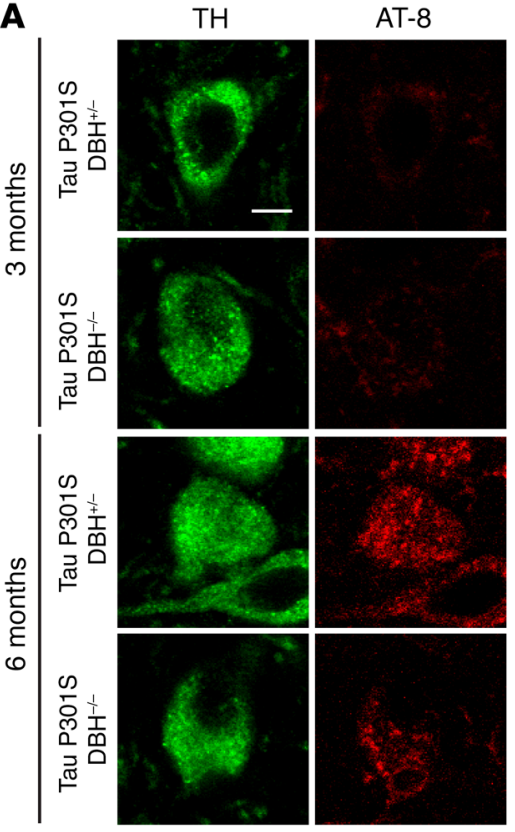

DAPI
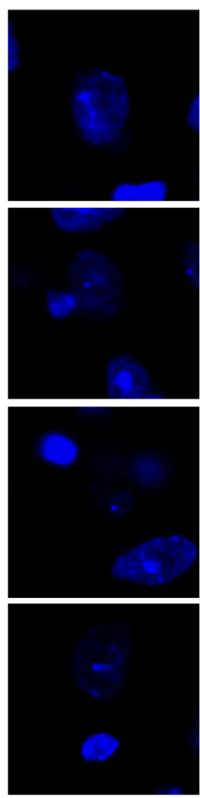

Merge
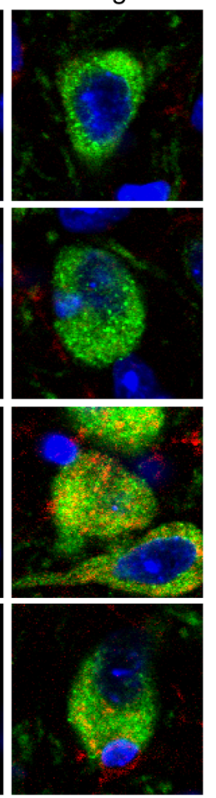

$\mathrm{TH}$
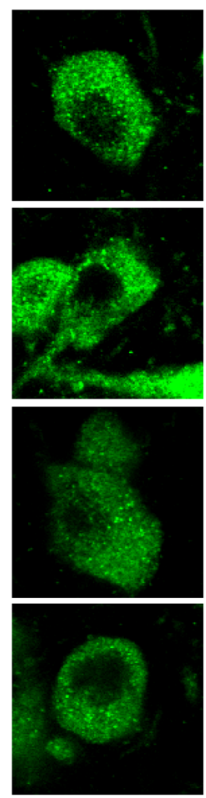

Tau N368
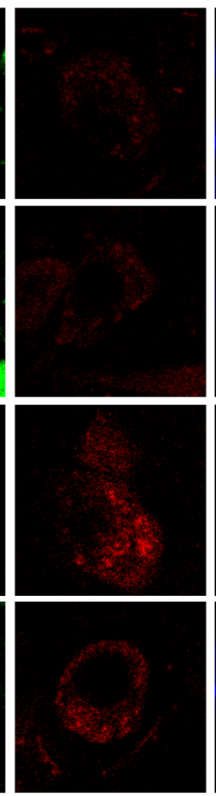

DAPI
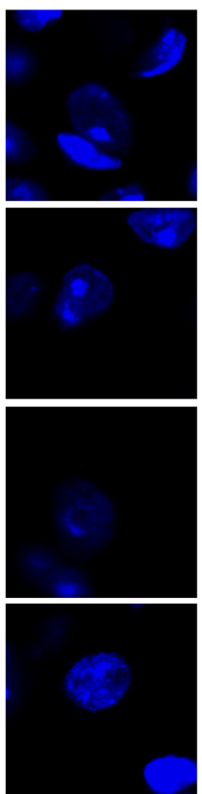

Merge
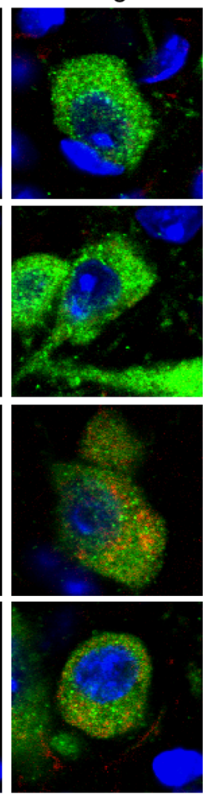

B

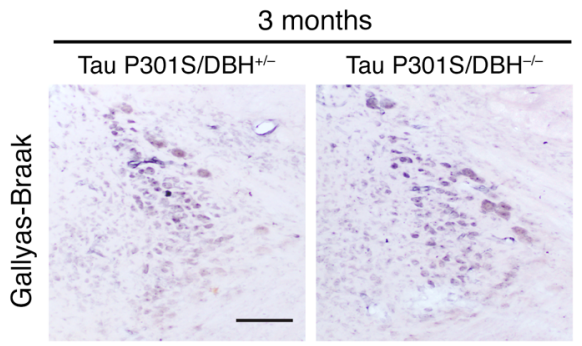

6 months

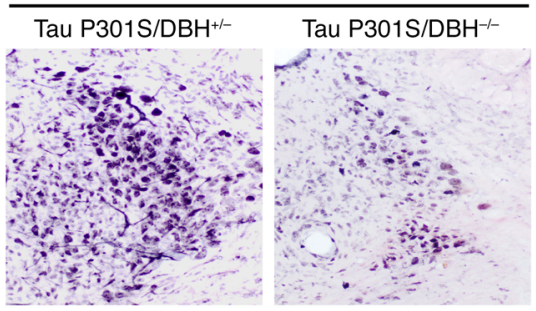

9 months

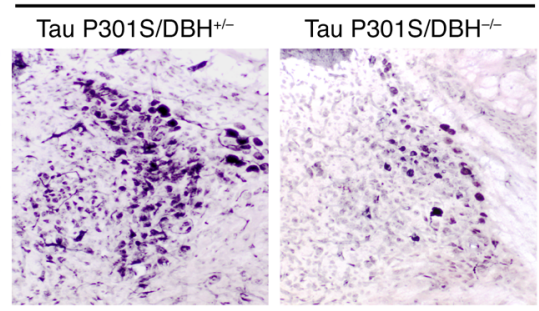

C

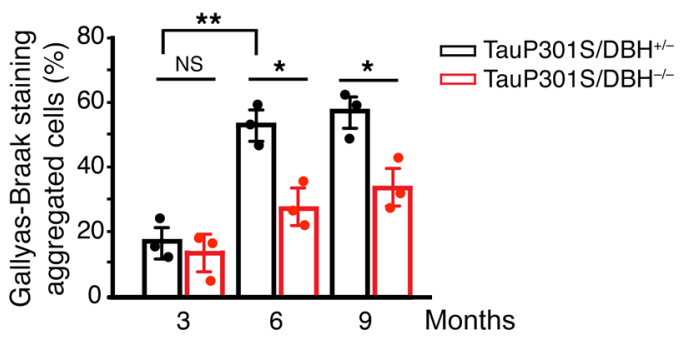

Figure 4. LC Tau pathology in P301S mice are dependent on NE. Tau P301S/DBH ${ }^{+/-}$ and Tau P3015/DBH ${ }^{-1}$ mice at various ages were examined for Tau pathology in the LC by immunohistochemistry. (A) Representative images of staining for TH (green), AT-8 or Tau N368 (red), and DAPI (blue). Scale bar: $20 \mu \mathrm{m}$. (B) Representative Gallyas-Braak (upper panels) staining. Scale bar: $100 \mu \mathrm{m}$. (C) Quantification of Gallyas-Braak staining. Data are shown as the mean \pm SEM ( $n=3$ per group). ${ }^{*} P<0.05$ and ${ }^{*} P<0.01$, by 2 -way ANOVA.

during a probe trial (Figure 5, C-E). Similar results were observed with cued and contextual fear conditioning, which test hippocampal-independent and -dependent associative learning and memory, respectively (Figure 5, F and G). AT8 and ThioS staining in the hippocampus also demonstrated that Tau pathology was increased age-dependently in Tau P301S/ $\mathrm{DBH}^{+-}$mice compared with Tau P301S/ $\mathrm{DBH}^{-/}$mice (Supplemental Figure 5D). Thus, the full expression of Tau pathology and cognitive impairment in Tau P301S mice depends on NE.

We next examined the impact of NE depletion on Tau pathology in the LC triggered by Tau cleavage. We reasoned that if DOPEGAL promotes the activation of AEP, then $\mathrm{DBH}^{-/}$mice that cannot produce DOPEGAL would be protected from Tau pathology. We injected AAV encoding human Tau P301S or AEP-resistant Tau P301S N255A/N368A into the $\mathrm{LC}$ of $\mathrm{DBH}^{+/-}$and $\mathrm{DBH}^{-/}$mice and conducted IF costaining with TH/AT8 and TH/Tau N368, as well as Gallyas-Braak silver staining. As expected, Tau P301S AAV elicited much greater Tau pathology than uncleavable Tau P301S N255A/368A virus. Strikingly, we found that the ability of Tau P301S to trigger AT8, N368, and Gallyas-Braak staining was attenuated significantly in $\mathrm{DBH}^{-/-}$mice (Supplemental Figure 5, A-C). These data suggest that NE promotes Tau P301S N368 cleavage by AEP and its subsequent phosphorylation and aggregation.

DOPEGAL is toxic to LC neurons in vivo. MAO-A and DOPEGAL are upregulated in the LC of postmortem AD brains (24) and DOPEGAL (but not NE or its other oxidative or O-methylated metabolites) is toxic to differentiated catecholaminergic PC12 cells (23). To explore whether DOPEGAL is toxic to LC neurons in vivo and the potential involvement of AEP and Tau, we infused DOPEGAL $(0.25 \mu \mathrm{g})$ into the LC of 2-month-old WT, Tau knockout $\left(\mathrm{Tau}^{-/}\right)$, MAPT transgenic mice that overexpress WT human Tau, and $\mathrm{MAPT} / \mathrm{AEP}^{-/-}$mice. Four days following DOPEGAL administration, IF costaining with TH and TUNEL revealed diminished $\mathrm{TH}$ and greater apoptosis in MAPT mice compared with WT, and 
A

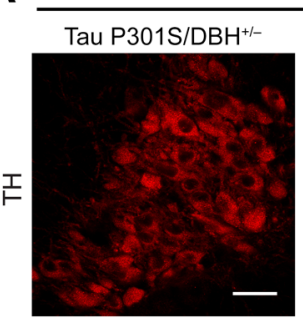

3 months

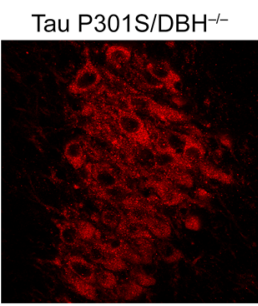

6 months

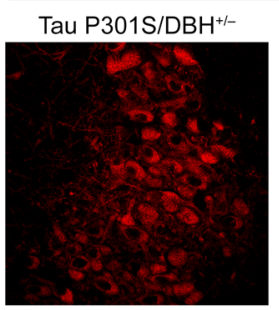

9 months
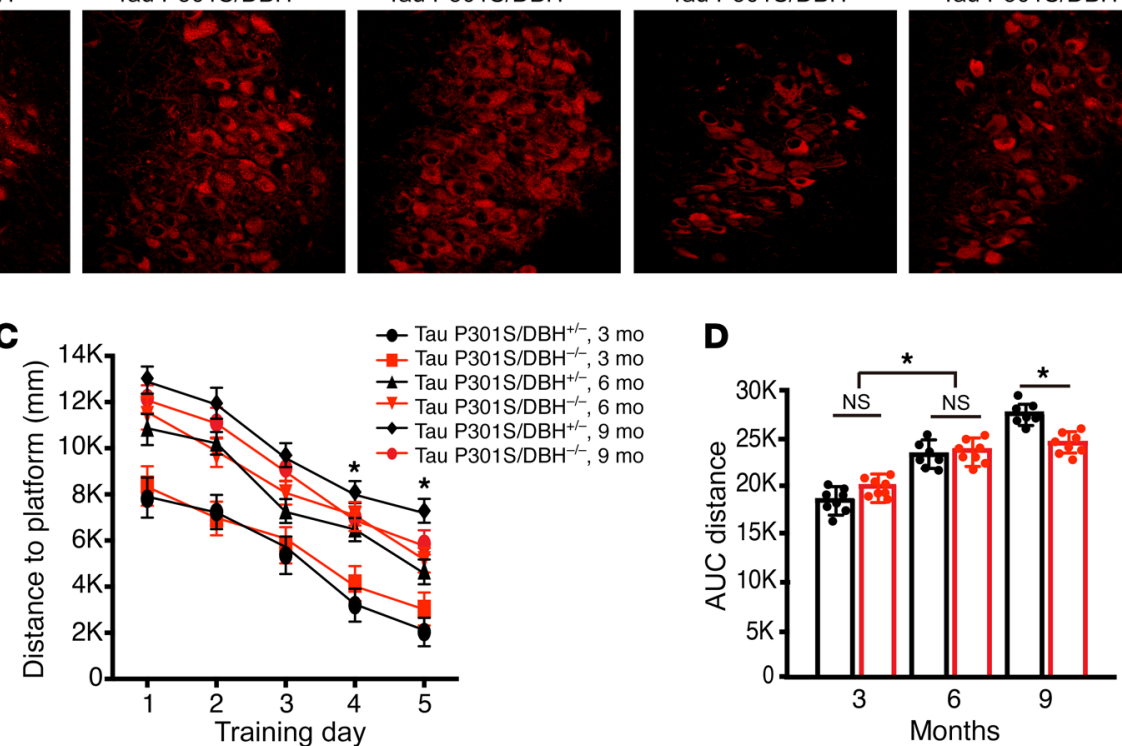
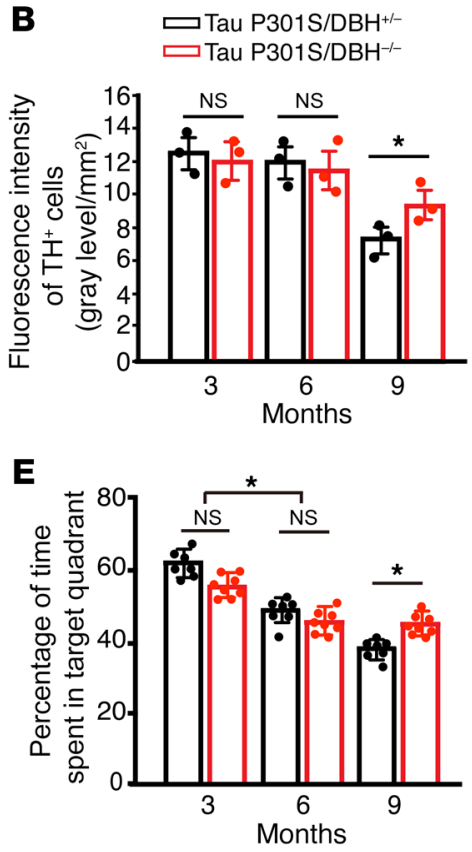

$\mathbf{F}$

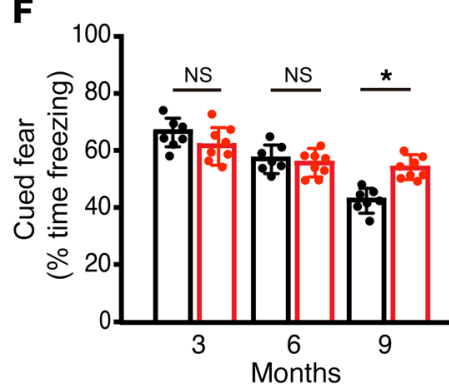

G

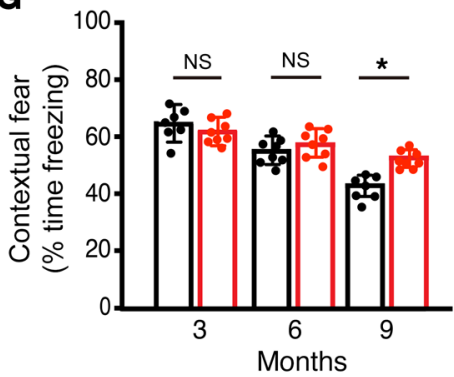

Figure 5. LC neurodegeneration and cognitive impairment in P3015 mice are dependent on NE. Tau P301S/DBH ${ }^{+/-}$and Tau P301S/DBH ${ }^{-/-}$mice at various ages were examined for neuronal cell death in the LC by immunohistochemistry and cognitive impairment using the MWM and fear conditioning test. (A) Representative images of TH staining. Scale bar: $100 \mu \mathrm{m}$. (B) Quantification of TH staining. Data are shown as the mean $\pm S E M\left(n=3\right.$ per group). ${ }^{*} P<0.05$, by 2-way ANOVA. (C) Distance traveled to the platform, (D) AUC for total distance traveled, and (E) percentage of time spent in the quadrant previously containing the platform during the probe trial in the MWM, and the percentage of time spent freezing during the (F) cued fear and (G) contextual fear tests following fear conditioning. All data were analyzed using 1-way ANOVA and are shown as the mean \pm SEM. $n=8$ per group. ${ }^{*} P<0.05$, by 2 -way ANOVA.

these effects were abrogated by Tau or AEP depletion (Figure 6, $\mathrm{A}-\mathrm{C})$. These results indicate that DOPEGAL-induced degeneration of LC neurons in vivo is partially mediated by a Tau- and AEP-dependent mechanism.

To investigate whether acceleration of endogenous NE metabolism and DOPEGAL production would have similar effects, we injected AAV-MAO-A or control virus into the LC of WT and MAPT mice and assessed pathology 3 months later. MAO-A overexpression failed to trigger neuronal loss in WT mice but significantly enhanced apoptosis and loss of TH-positive cells in MAPT mice (Figure 7, A-C), which was accompanied by AT8 and Tau N368 staining in LC neurons (Figure 7E). We examined the levels of catecholamines and their metabolites in the LC by HPLC and verified that MAO-A overexpression significantly increased DOPEGAL and tended to decrease NE, as expected, without affecting any other analyte (Figure 7D). The staining of AT8 and Thios in the hippocampus also demonstrated that Tau pathology was increased by MAO-A overexpression in MAPT mice compared with WT mice (Supplemental Figure 5E). MWM and fear conditioning tests demonstrated that MAO-A triggered significant cognitive dysfunction in MAPT mice compared with control virus, whereas it had no effect on WT mice (Figure 7, F-J). These data suggest that Tau exacerbates DOPEGAL-mediated LC neuron toxicity and cognitive impairment.

LC-derived human Tau can spread to interconnected brain regions in MAPT and $3 x \mathrm{Tg}$ mice. Because the $\mathrm{LC}$ is the first brain structure to develop Tau lesions and has widespread connections to other areas of the brain, and Tau is capable of trans-synaptic propagation, LC neurons have been proposed to be the critical initiators of the stereotypical spread of Tau pathology in $\operatorname{AD}(4,5)$. To explore this idea, we injected 3-month-old MAPT mice with either AAV-mCherry alone or AAV-mCherry + AAV-Tau under control of the noradrenergic-specific PRSx8 promoter into the LC (34), and then monitored Tau pathology throughout the brain 3 months later (Figure 8A). mCherry (red) and TH (blue) IF costaining confirmed viral targeting to noradrenergic LC neurons, and AT8 (green) and N368 (green) Tau immunoreactivity were evident in the mice that received AAV-mCherry/AAV-Tau, but not mCherry alone (Figure 
A

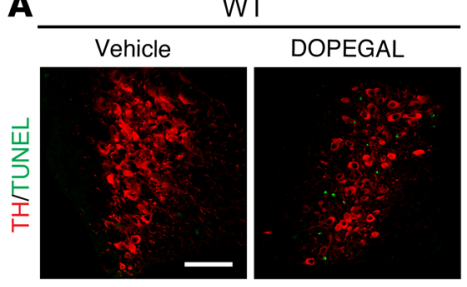

MAPT $^{-1-}$

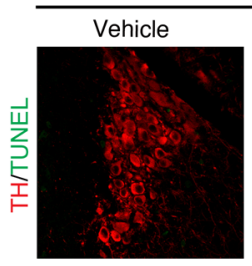

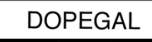

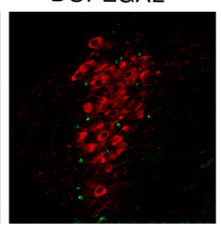

MAPT

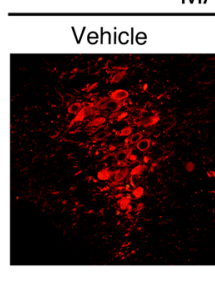

MAPT/AEP-1-

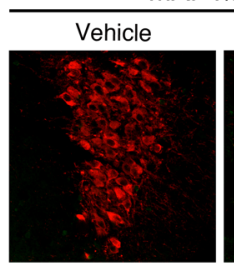

DOPEGAL $(0.25 \mu \mathrm{g})$

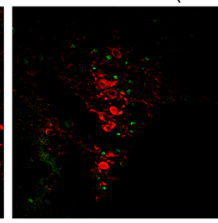

DOPEGAL

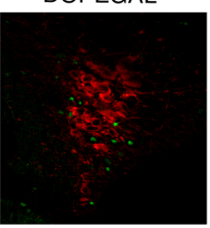

B

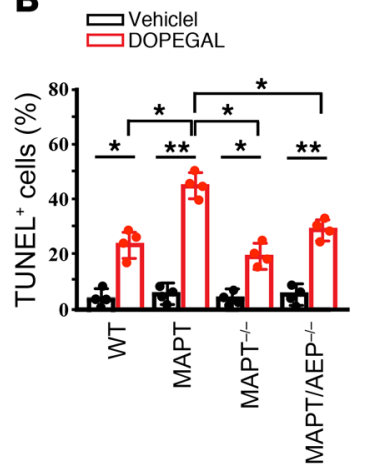

C

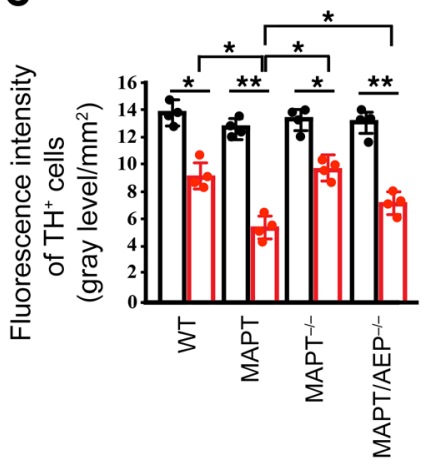

Figure 6. DOPEGAL injection into the LC induces cell death dependent on Tau and AEP. DOPECAL (0.25 $\mu$ g) was injected into the LC regions of WT, MAPT $^{-/-}$, MAPT, or MAPT/AEP ${ }^{-/-}$mice. (A) Representative images of TH (red) and TUNEL (green) staining in the LC 4 days following DOPEGAL injection. Scale bar: $100 \mu \mathrm{m}$. (B) Quantification of TUNEL ${ }^{+}$cells. (C) Quantification of TH intensity. Data are shown as the mean \pm SEM. $n=4$ per group. ${ }^{*} P<0.05$ and ${ }^{*} P<0.01$, by 2 -way ANOVA.

8B). AT8 IHC revealed that aberrant Tau had spread from the LC to the cerebellum, midbrain, hippocampus, entorhinal $\mathrm{Cx}$, and $\mathrm{Cx}$ (Figure 8A). Moreover, IF and Gallyas-Braak staining indicated that hyperphosphorylated Tau, AEP-cleaved Tau, and aggregated Tau were present in the entorhinal $\mathrm{Cx}$ (EC), hippocampus (HC), and $\mathrm{Cx}$ of AAV-Tau injected mice (Figure 8C and Figure 9A). By contrast, neither aberrant Tau nor mCherry were detected in the forebrain of mice injected with AAV-mCherry alone (Figure 8C), indicating that Tau pathology, rather than the virus itself, spread from the LC to these distal regions. Similar results were obtained when we performed the identical experiment in 3xTg mice (Supplemental Figure $6 \mathrm{~A}-\mathrm{C}$ ).

To test whether pathogenic Tau originating in the LC can induce cognitive deficits, we conducted MWM and fear conditioning 3 months after intra-LC Tau virus administration in MAPT and $3 \times \mathrm{Tg}$ mice. Tau-injected mice spent significantly less time in the target quadrant during the MWM probe trial (Figure 9, B-D) and were impaired in both cued and contextual freezing following fear conditioning (Figure 9, E and F). Together, these behavioral tests indicate that LC-derived Tau pathology can spread to the forebrain and produce cognitive impairment.

AEP cleavage of Tau increases its aggregation and neurotoxicity, and depletion of AEP diminishes Tau pathology in Tau P301S mice (18). Tau cleavage by AEP is necessary and sufficient for its neurotoxicity in LC neurons (Supplemental Figure 2), and the appearance of AEP-cleaved Tau N368 is tightly associated with AT8 immunoreactivity, suggesting that AEP cleavage of Tau may facilitate its spread. We prepared primary LC neurons from neonatal WT and $\mathrm{AEP}^{-/-}$mice and infected them with AAV-PRSx8-Tau or control virus. IF costaining with TH and Tau5 or the humanspecific Tau HT7 antibody showed that human Tau was specifically overexpressed in the LC neurons (Supplemental Figure 7A, upper panels). TH and N368 or AT8 costaining demonstrated that human Tau was truncated at N368 and hyperphosphorylated in WT LC neurons, whereas both N368 and AT8 immunoreactivity were attenuated in $\mathrm{AEP}^{-/-} \mathrm{LC}$ neurons (Supplemental Figure 7A, lower panels). To determine whether AEP is necessary for Tau aggregation and spread in vivo, we injected 3-month-old WT and $\mathrm{AEP}^{-/}$ mice with AAV-PRSx8-Tau + AAV-PRSx8-mCherry in the LC and monitored Tau pathology in the LC and forebrain 3 months later. AT8, N368 Tau, and Gallyas-Braak staining indicated that hyperphosphorylated and AEP-cleaved Tau accumulation in the LC and subsequent spread to the $\mathrm{HC}, \mathrm{Cx}$, and $\mathrm{EC}$ were largely retarded in $\mathrm{AEP}^{-/-}$mice (Figure 10, $\mathrm{A}$ and $\mathrm{B}$, and Figure 11A). A schematic map of Tau pathology spread from the LC in MAPT, 3xTg, WT, and $\mathrm{AEP}^{-/-}$ mice as viewed by AT8 staining is shown in Supplemental Figure 7B. Moreover, deletion of AEP prevented cognitive impairment induced by Tau overexpression in the LC (Figure 11, B-F). These data indicate that AEP contributes to the spread of LC-derived Tau pathology to the forebrain and associated cognitive deficits.

\section{Discussion}

Pathogenic forms of Tau first appear in noradrenergic LC neurons (3-6), which degenerate later in $\mathrm{AD}$, and LC dysfunction contributes to $\mathrm{AD}$-like neuropathology and cognitive deficits $(6,8,9,11-13$, 16). However, the mechanism underlying the selective vulnerability of LC neurons has remained elusive. In the current study, we report that DOPEGAL, the NE metabolite produced by MAO-A oxidation, triggers AEP activation via oxidative stress, resulting in Tau N368 cleavage that is prone to hyperphosphorylation, aggregation, and propagation, and triggers noradrenergic neuronal death. In addition, DOPEGAL, but not NE, facilitates the fibrilization of purified recombinant Tau. Tau hyperphosphorylation and N368 cleavage were age-dependently increased in the LC of both MAPT and 3xTg mice, consistent with our previous finding that AEP is upregulated and activated in the brain during aging. Importantly, we made the same observations in the LC neurons of human AD brains, indicating that active AEP contributes to Tau pathology in the LC.

DOPEGAL is an appealing candidate to explain the selective vulnerability of LC neurons in AD for several reasons. First, it is only produced in noradrenergic cells. Second, it is increased in the LC of human AD brains and is toxic to noradrenergic neurons. 

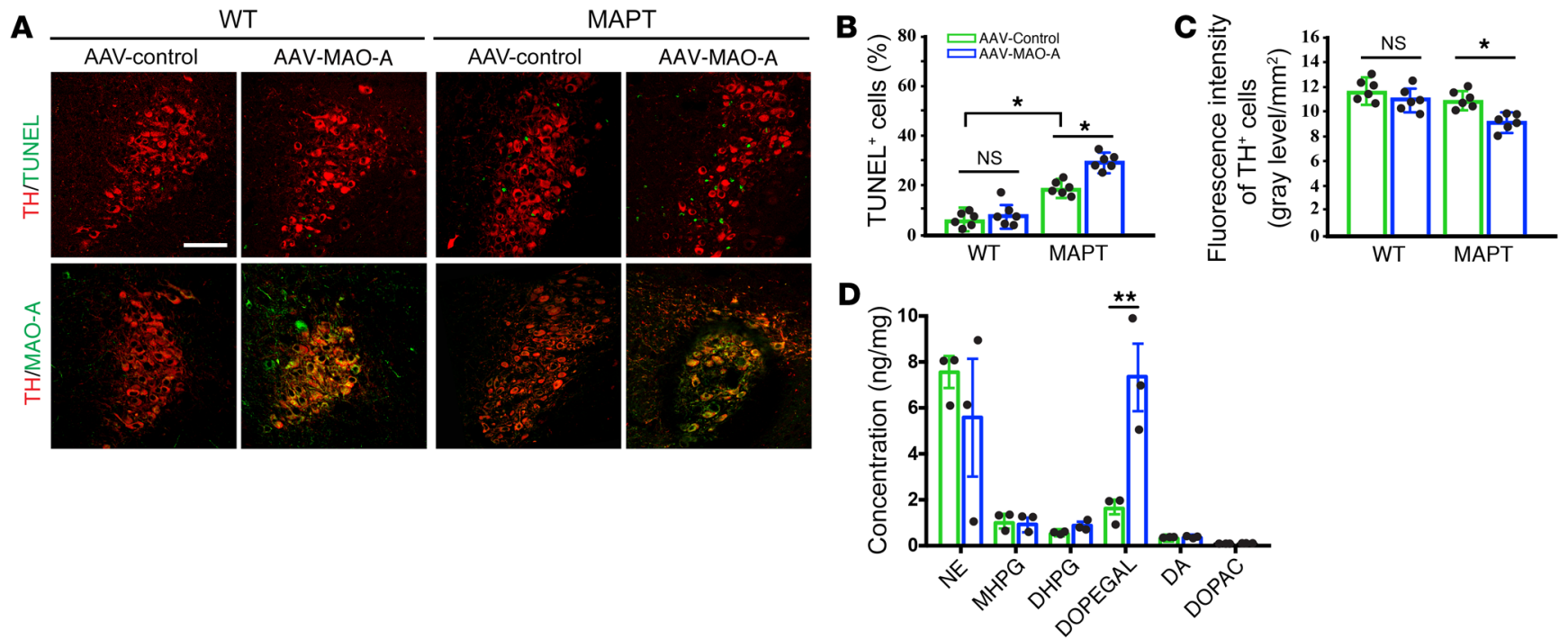

E
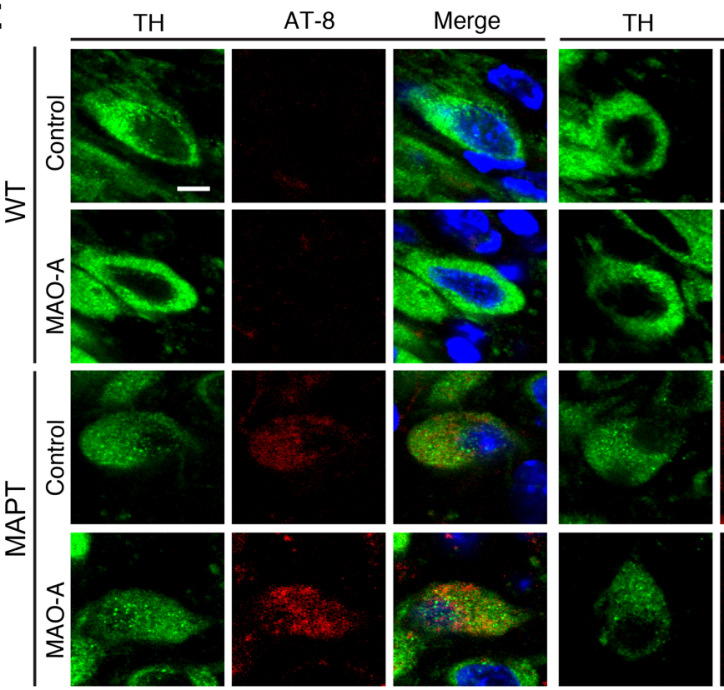

Tau N368
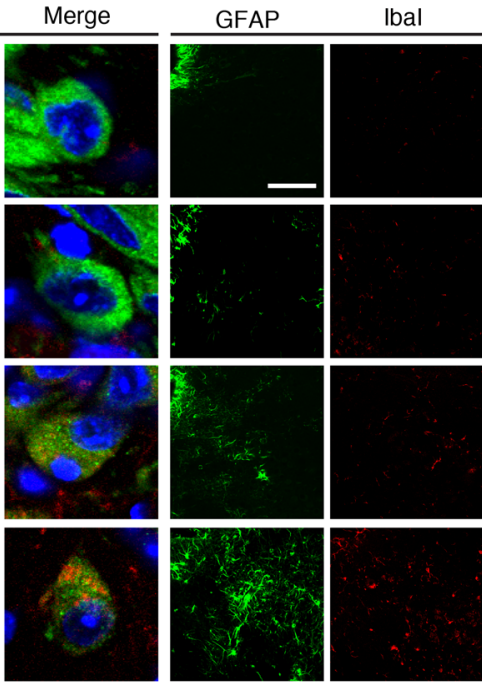

Merge Gallyas-Braak
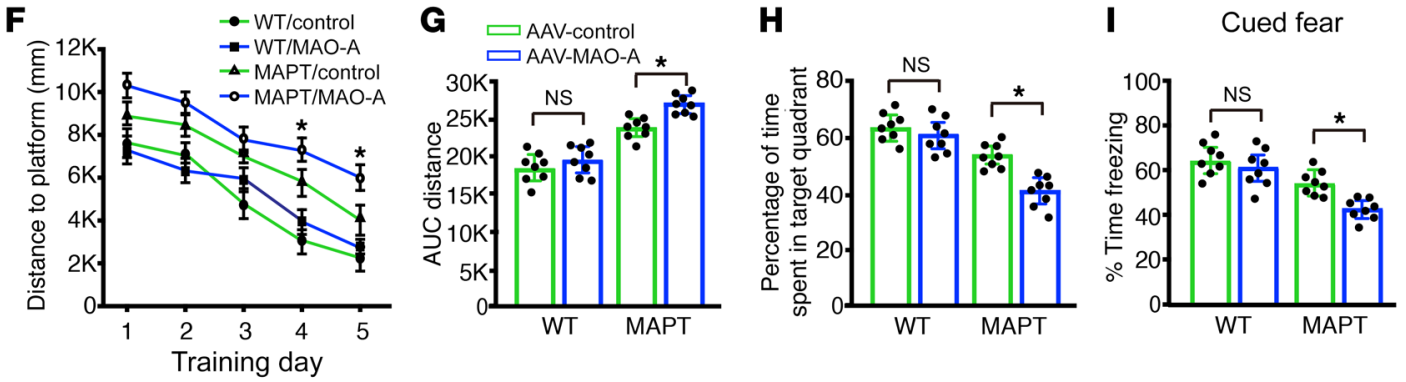

J Contextual fear

Figure 7. Increasing DOPEGAL in the LC by MAO-A overexpression induces Tau pathology, cell death, and cognitive impairment in MAPT transgenic mice. AAV-MAO-A was injected into LC regions of WT or MAPT mice. (A) Representative images of TH (red) and TUNEL (green) staining in the LC 3 months following AAV-MAO-A injection. Scale bar: $100 \mu \mathrm{m}$. (B) Quantification of TUNEL. (C) Quantification of TH intensity. Data are shown as the mean \pm SEM. $n=6$ per group. ${ }^{*} P<0.05$, by 2 -way ANOVA. (D) Measurement of DOPEGAL in the LC of AAV-MAO-A-injected WT mice via HPLC analysis. Data were analyzed using 2-way ANOVA and are shown as the mean \pm SEM ( $n=3$ per group). ${ }^{* *} P<0.01$, by 2 -way ANOVA. (E) Representative images of staining for TH (green), AT-8 or Tau N368 (red), and DAPI (blue). Right panels are representative images of Gallyas-Braak staining. Scale bars: $20 \mu \mathrm{m}$ and $100 \mu \mathrm{m}$, respectively. (F-J) Distance traveled to the platform (F), AUC for total distance traveled (G), and percentage of time spent in the quadrant $(\mathbf{H})$ previously containing the platform during the probe trial in the MWM, and percentage of time spent freezing during the cued fear (I) and contextual fear $(\mathrm{J})$ tests following fear conditioning. All data were analyzed using 1-way ANOVA and are shown as the mean \pm SEM. $n=8$ per group. ${ }^{*} P<0.055$, by 1 -way ANOVA. 
A

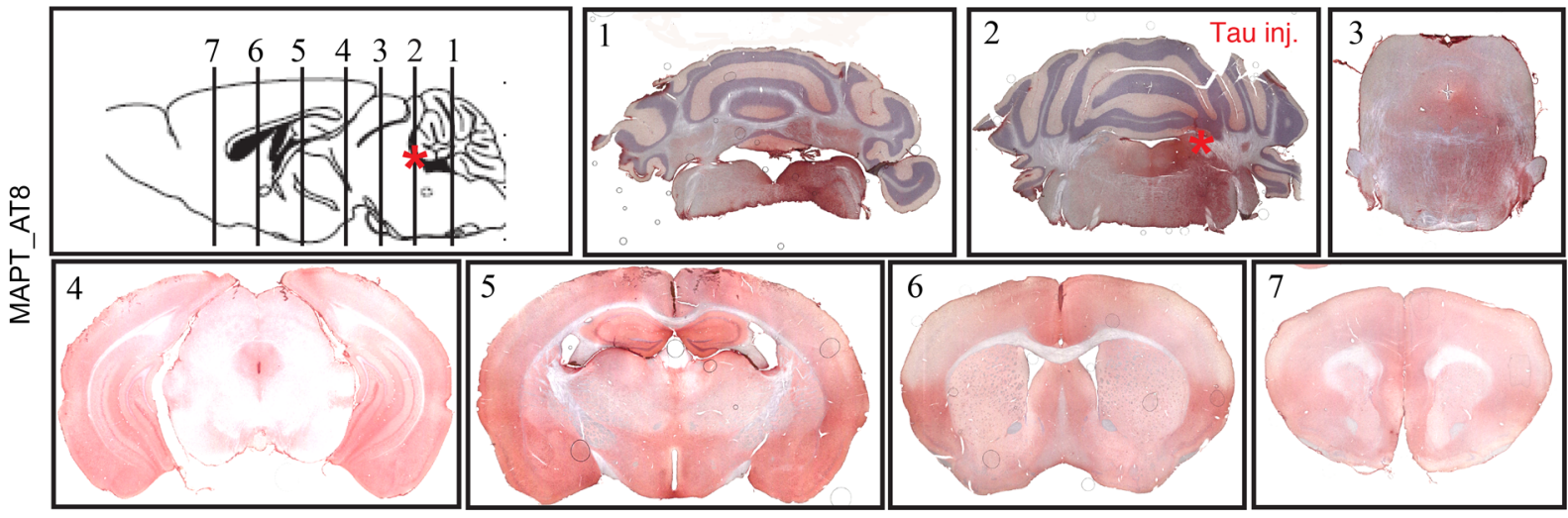

B

AAV-control (mCherry)

AAV-Tau (mCherry)
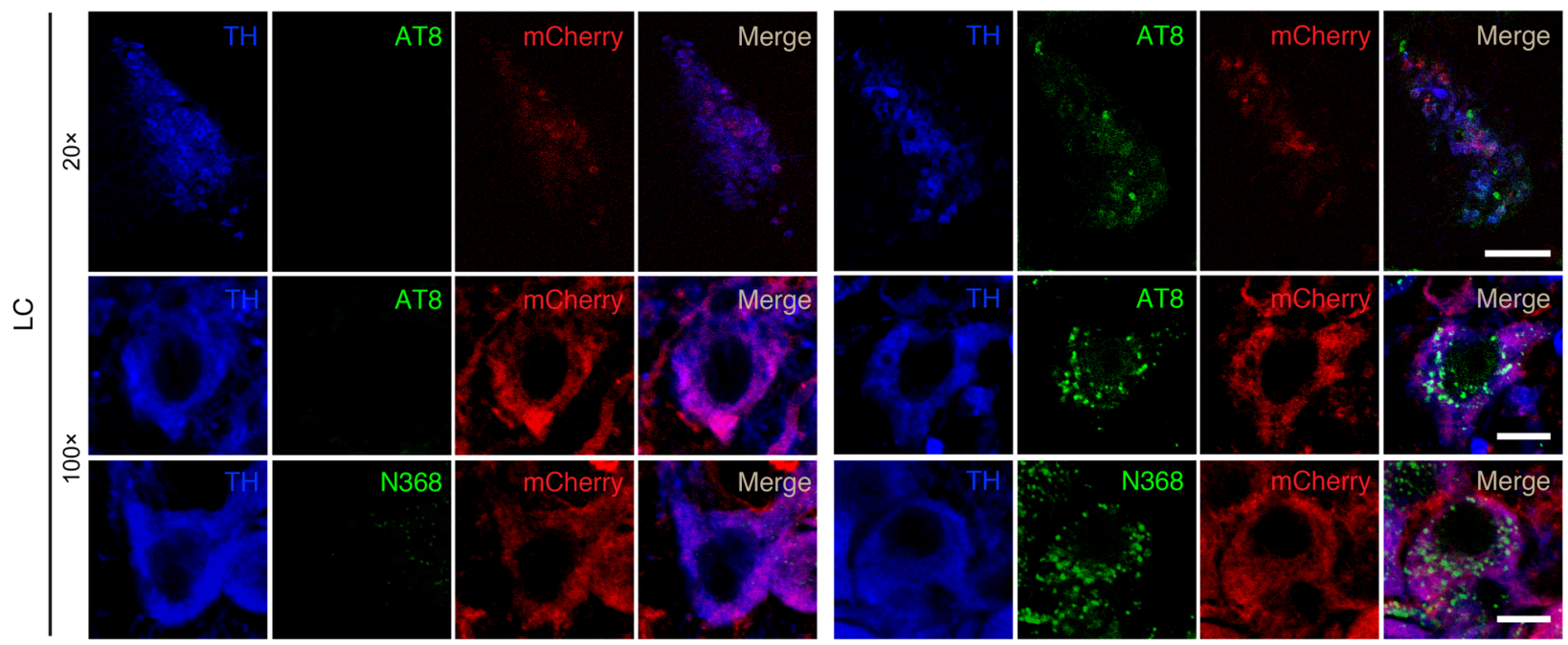

C

AAV-control (mCherry)

AAV-Tau (mCherry)
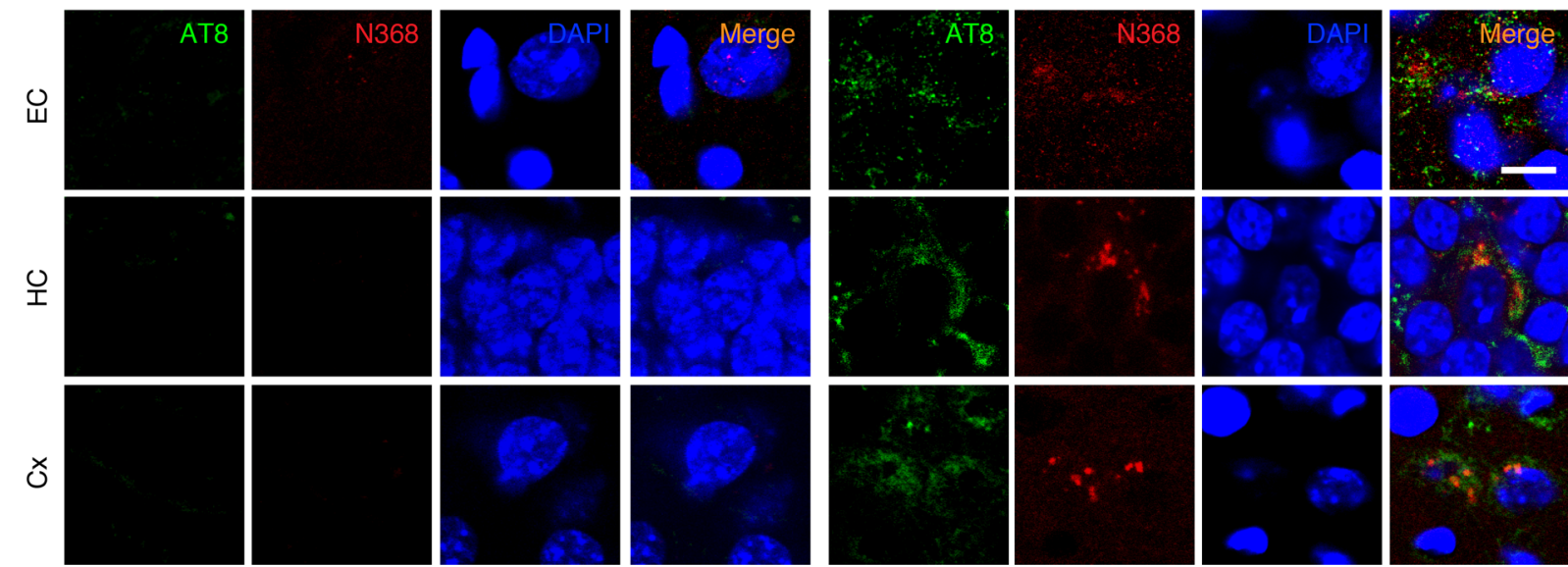

Figure 8. Viral-mediated Tau expression in the LC drives propagation of pathology to the forebrain in MAPT transgenic mice. LC-specific AAV-PRS 8 Tau + AAV-PRSx8-mCherry, or AAV-PRSx8-mCherry alone were injected into the LC of MAPT mice, and mice were assessed for Tau pathology throughout the brain by immunohistochemistry 3 months later. (A) Representative images of AT8 immunostaining throughout the brain of MAPT mice. (B) Representative images of immunofluorescent staining for TH (blue), AT8 or Tau N368 (green), and mCherry (red) in the LC of MAPT mice. Scale bar: $100 \mu \mathrm{m}$ (top panels) and $20 \mu \mathrm{m}$ (bottom panels). (C) Representative images of immunofluorescent staining for AT8 (green) or Tau N368 (red), and DAPI (blue) in the EC, $H C$, and frontal Cx of MAPT mice. Scale bar: $20 \mu \mathrm{m}$. 
A
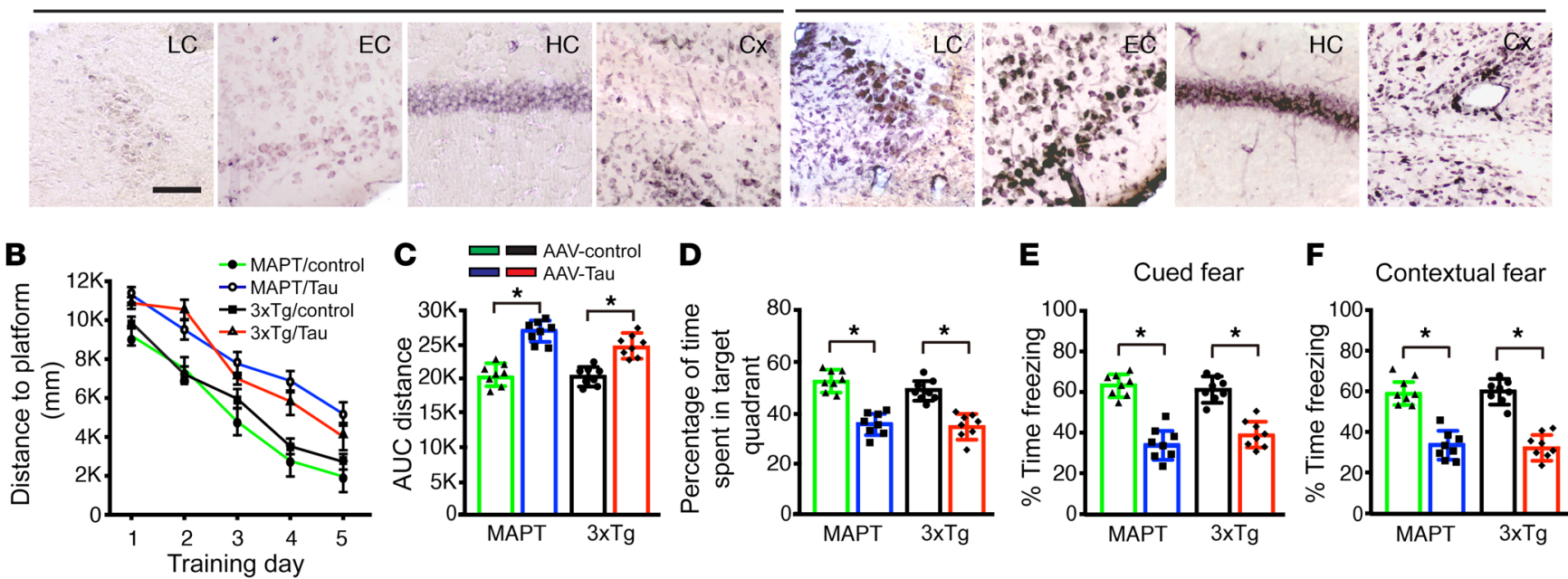

Figure 9. Viral-mediated Tau expression in the LC induces Tau fibrilization and cognitive impairment in MAPT and 3XTC mice. AAV-Tau or AAV-mcherryinjected mice were assessed for Tau fibrilization by Gallyas-Braak staining and cognitive impairment using MWM and fear conditioning test. (A) Representative Gallyas-Braak staining in the LC, EC, HC, and Cx of MAPT mice. Scale bar: $100 \mu \mathrm{m}$. (B) Distance traveled to the platform, (C) AUC for total distance traveled, and (D) percentage of time spent in the quadrant previously containing the platform during the probe trial in the MWM, and percentage of time spent freezing during the (E) cued fear and (F) contextual fear tests following fear conditioning in MAPT and 3xTg mice. All data were analyzed using 1-way ANOVA and are shown as the mean \pm SEM. $n=8$ per group. ${ }^{*} P<0.05$ compared with the control group.

Finally, the close neurochemical relationship between NE and DA suggests that the toxicity of the DA MAO metabolite DOPAL may have a correlate in the noradrenergic system. Previous studies show that physiological concentrations of DOPAL trigger the formation of $\alpha$-Syn oligomers and aggregates in both a cell-free system and in cell culture, and produce cytotoxicity in vitro and in vivo. We recently reported that DOPAL activates AEP in dopaminergic neurons and leads to $\alpha$-synuclein N103 cleavage by AEP, resulting in its aggregation and dopaminergic neuronal degeneration in the SN (21). Thus, DOPAL- $\alpha$-Syn interactions may underlie the selective vulnerability of DA neurons in PD (35-39), and we suspected that a similar interaction might occur between DOPEGAL and Tau in AD. Here, we present multiple converging lines of evidence implicating DOPEGAL and AEP in the vulnerability of LC neurons to Tau pathology and toxicity. First, DOPEGAL, but not NE itself, increased AEP activity and cell death. Second, overexpression of MAO-A, which oxidizes NE into DOPEGAL, facilitated AEP activity, Tau N368 cleavage, and cell death, whereas decreasing MAO activity or blocking Tau cleavage had the opposite effect. Third, preventing DOPEGAL production via DBH depletion or Tau cleavage by $\mathrm{AEP}^{-/-}$mitigated the deleterious effects of Tau on LC neurons, whereas preventing the breakdown of DOPEGAL by AR or ALDH inhibition escalated AEP activity and cell death. Finally, full DOPEGAL toxicity was dependent on the presence of Tau. Together, these data suggest that DOPEGAL stimulates AEP activation via oxidative stress, resulting in Tau N368 cleavage and aggregation, LC neuronal degeneration, and the spread of Tau pathology to the forebrain and associated cognitive impairment (Figure 12). Given that DOPAL can directly modify $\alpha$-Syn, it is possible that DOPEGAL may covalently modify the lysine residues and oxidize Met groups on Tau, thus facilitating its conformational change and fibrillization. We did find that DOPEGAL accelerated the aggregation of recombinant Tau in vitro, but fur- ther experiments will be required to pinpoint the covalent and/or noncovalent mechanisms.

MAO-A generates $\mathrm{H}_{2} \mathrm{O}_{2}$ and DOPEGAL, when it metabolizes NE. Both $\mathrm{H}_{2} \mathrm{O}_{2}$ and DOPEGAL induce AEP activation and trigger cell death, and we have shown that the transcription factor, CEBP- $\beta$, regulates the expression of AEP (40). Conceivably, there is positive feedback between NE metabolism and CEBP- $\beta$, whereby increased levels of MAO-A and $\mathrm{H}_{2} \mathrm{O}_{2}$ activate CEBP- $\beta$, which, in turn, induces further MAO-A and AEP expression. MAO-A preferentially oxidizes serotonin and NE, whereas MAO-B preferentially oxidizes phenylethylamine, and both forms can oxidize DA (41). MAO-A oxidizes NE into DOPEGAL within less than 24 hours in SH-SY5Y cells (Supplemental Figure 2D). However, MAO-A overexpression by AAV infection is an indirect experiment to activate DOPEGAL in vivo, whereas DOPEGAL treatment is a direct approach to investigate DOPEGAL's acute toxicity to LC neurons. AAV takes several weeks to reach steady-state gene expression, and the increased oxidation of NE to more DOPEGAL is presumably gradual, because other enzymes are present in the LC that also metabolize DOPEGAL as well (i.e., aldehyde dehydrogenase and aldehyde reductase). The acute infusion of DOPEGAL likely overwhelms the normal machinery, leading to rapid toxicity, whereas the gradual production and buildup of DOPEGAL via AAV-MAO-A is likely to take more time. MAO-A is predominantly found in catecholaminergic neurons, with the highest concentration in the LC (42). Moreover, DOPEGAL and MAO-A levels are elevated 2.8- and 3.6-fold in AD LC neuronal cell bodies compared with controls (24), whereas MAO-B expression and activity are higher in multiple brain regions and cell types in $\mathrm{AD}(43,44)$. The preference of MAO-A for NE and the enriched LC expression suggest that MAO-A is the main driver of DOPEGAL production in vivo, although both MAO-A and MAO-B were capable of increasing AEP activity, Tau N368 cleavage, and cell death in our experiments. We have previously demonstrated that MAO-B plays a greater role in 
A

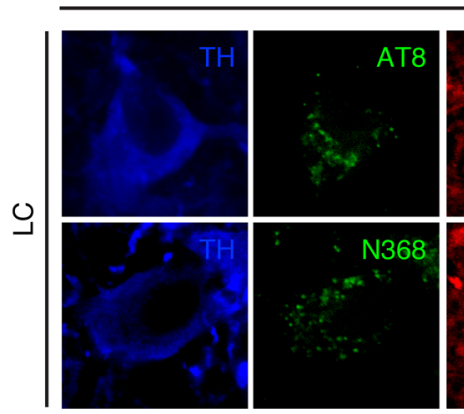

B

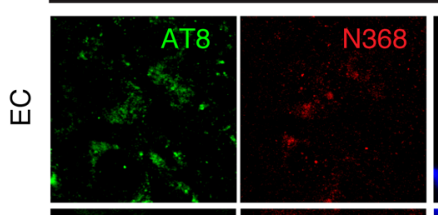

오

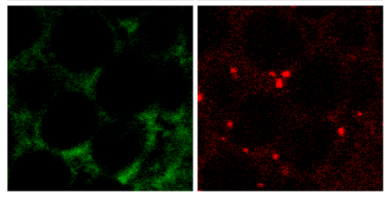

త

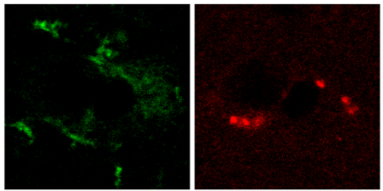

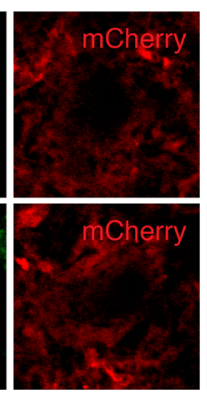
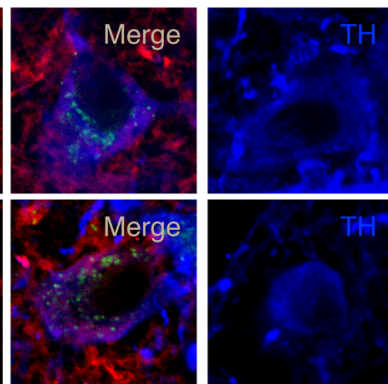

AEP KO AAV-Tau/mCherry
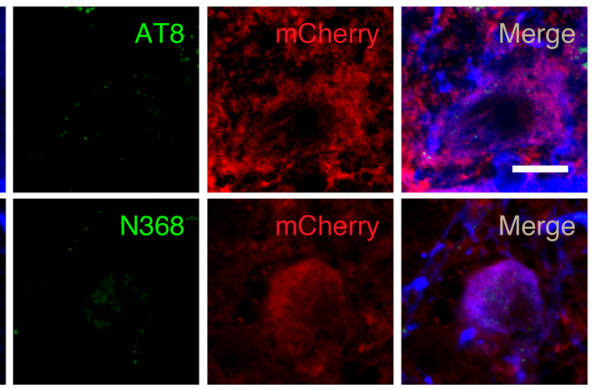

AEP KO_AAV-Tau/mCherry
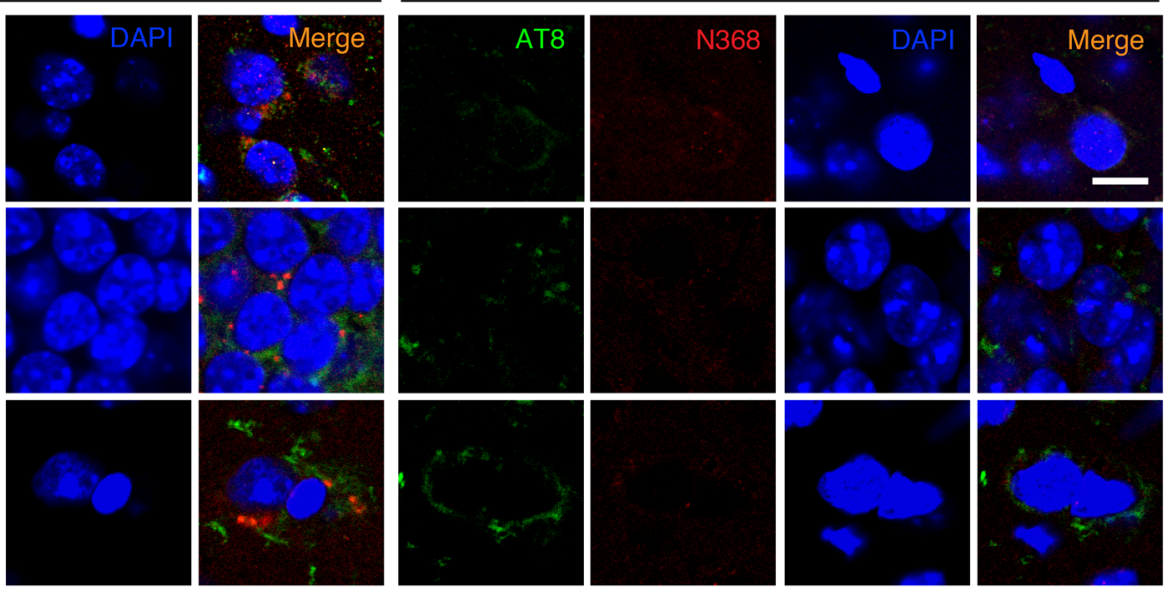

Figure 10. Viral vector-mediated Tau pathology in the LC and its propagation to the forebrain are blunted in AEP-/- mice. LC-specific AAV-PRSX8-Tau + AAV-PRSx8-mCherry were injected into the $L C$ of $\mathrm{AEP}^{+/+}$or $\mathrm{AEP}^{-/-}$mice, and pathology were assessed 3 months later. (A) Representative images of immunofluorescence staining of TH (blue), AT8 or N368 (green), and mCherry (red) in the LC. Scale bar: $20 \mu \mathrm{m}$. (B) Representative images of immunofluorescent staining of AT8 (green), N368 (red), and DAPI (blue) in the EC, HC, and Cx. Scale bar: $20 \mu \mathrm{m}$.

generating the neurotoxic DA metabolite DOPAL in PD $(21,45)$, and here we show that NE metabolism into DOPEGAL by MAO-A in the progression of $\mathrm{AD}$-like pathology. Combined, these results support our model that DA metabolism into DOPAL and MAO-A-mediated conversion of NE into DOPEGAL induce $\alpha$-synuclein toxicity in SN and Tau toxicity in LC, respectively, leading to the selective vulnerability of catecholamine neurons in PD and AD (Figure 12).

Because DOPEGAL, but not NE itself, generates free radicals and activates mitochondrial permeability transition (27), DOPEGAL may contribute to the loss of noradrenergic LC neurons in $\mathrm{AD}$ (46). Employing $\mathrm{Tau}^{-/-}$neurons and mice, we showed that DOPEGAL-induced neurotoxicity is partially dependent on Tau. Knockout of AEP significantly ameliorated the LC neuronal degeneration and Tau pathology elicited by DOPEGAL exposure in MAPT mice, indicating that AEP cleavage of Tau is an important factor in LC vulnerability. This finding is further supported by our experiments with the AEP cleavage-resistant Tau P301S/ N255/368A mutant. In SH-SY5Y cells, we found that the ability of AEP-cleaved Tau N368 to trigger cell death was more robust than WT Tau and comparable with P301S Tau. By contrast, introducing the N255/368A mutations onto a P301S background substantially reduced the toxicity of P301S Tau. Moreover, P301S Tau strongly induced Tau pathology in LC neurons in vivo that was associated with Tau N368 cleavage, while AEP-resistant P301S/N255/368A
Tau was much less active. P301S-induced Tau pathology was also attenuated in the $\mathrm{LC}$ of $\mathrm{DBH}^{-/-}$mice, indicating that $\mathrm{NE}$ is required for Tau to exert its full neurotoxicity. This interpretation is further supported by our findings that siRNA-mediated knockdown of DBH (and thus NE) production blunted Tau-induced cell loss in catecholaminergic SH-SY5Y cells. Combined, these data support a model whereby LC vulnerability in AD is caused by NE metabolism to DOPEGAL by MAO-A, which activates AEP and Tau cleavage, leading to Tau pathology and neuronal death. The Braak and Grinberg groups reported that hyperphosphorylated Tau emerges first in LC (Braak stage 0), but frank degeneration of LC neurons is not evident until Braak stage 3 to $4(3,6)$, although we have shown that pathogenic Tau induces LC fibers/terminals/NE loss before cell body death in animal models $(11,14)$. The reasons for the delayed cell death are not clear, but conceivably, it takes time for the cellular stress and damage to reach the levels necessary to activate AEP, triggering Tau cleavage and fatal toxicity.

Previous research has shown that Tau pathology is detectable in $3 x T g$ mice at 9 to 12 months (47) and in MAPT mice at 12 months (48), so in our experiments, any appearance of aberrant Tau at 6 months must be derived from the LC. We observed the appearance of hyperphosphorylated, AEP-cleaved, and Gallyas-Braak-positive forms of Tau in the EC, HC, and Cx of both transgenic strains following intra-LC infusion of mCherry + Tau virus. Importantly, 
A

3

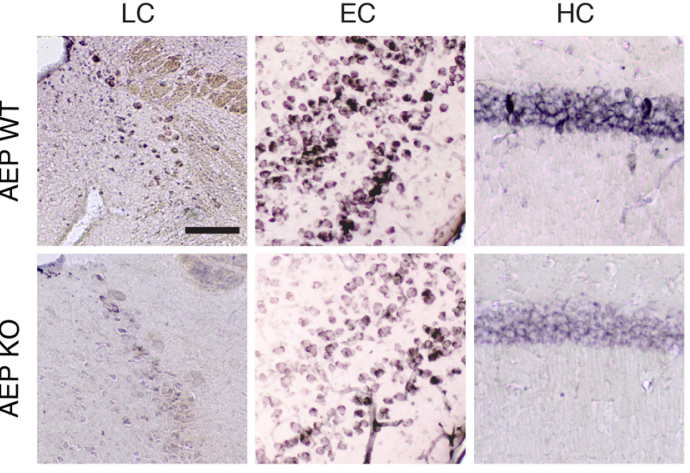

Cx

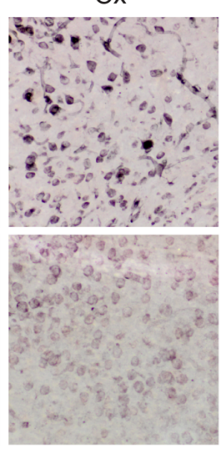

B

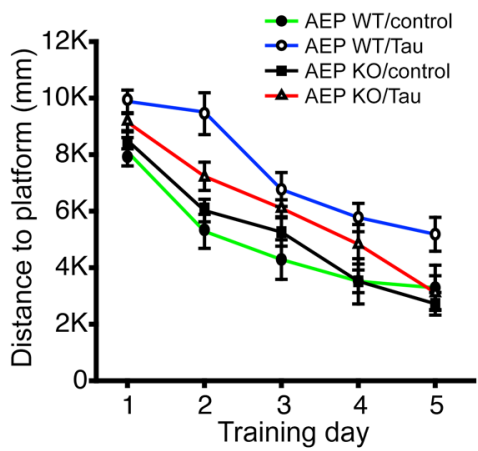

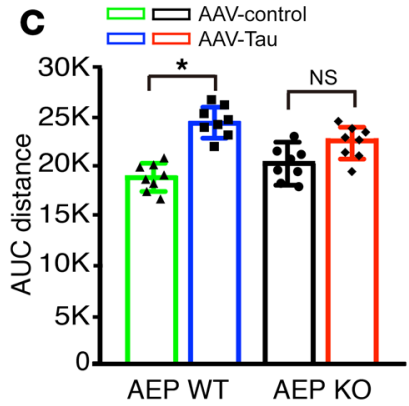

D

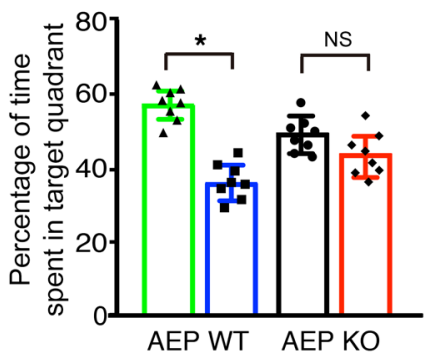

E

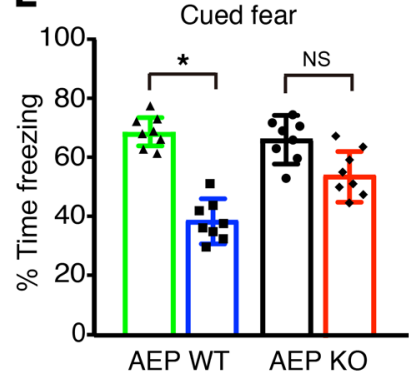

$\mathbf{F}$

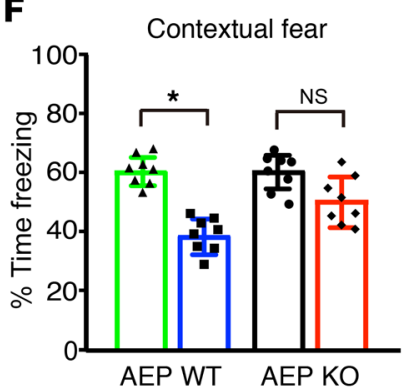

Figure 11. Viral vector-mediated Tau fibrilization and cognitive impairment are alleviated in AEP- $/$ - mice. LC-specific AAV-PRSx8-Tau + AAV-PRSx8mCherry were injected into $\mathrm{LC}$ of $\mathrm{AEP}^{+/+}$or $\mathrm{AEP}^{-/-}$mice, and fibrilization and cognitive impairment were assessed 3 months later. (A) Representative Gallyas-Braak staining in the LC, EC, HC, and Cx of AEP ${ }^{+/+}$or AEP ${ }^{-/-}$mice. Scale bar: $100 \mu m$. (B) Distance traveled to the platform, (C) AUC for total distance traveled, and (D) percentage of time spent in the quadrant previously containing the platform during the probe trial in the MWM, and percentage of time spent freezing during the (E) cued fear and (F) contextual fear tests following fear conditioning. All data were analyzed using 1-way ANOVA and are shown as the mean \pm SEM. $n=8$ per group. ${ }^{*} P<0.05$ compared with the control group.

mCherry did not spread to distal brain regions, and there was no forebrain Tau pathology detected in mice that received mCherry alone. These results clearly show that, at least in these models, Tau pathology originating in the LC is capable of propagating to interconnected brain regions, including those that also show early vulnerability in $\mathrm{AD}$. To determine whether this spread of pathogenic Tau had any functional effects, we tested mice for signs of cognitive impairment and found that the performances of $3 \mathrm{xTg}$ and MAPT mice in the MWM and fear conditioning assays worsened following Tau expression in the LC and subsequent spread to the forebrain. Remarkably, both the spread of Tau pathology and the accelerated cognitive deficits following Tau expression in the $\mathrm{LC}$ were alleviated in $\mathrm{AEP}^{-/-}$mice. Here, we provide a combination of in vitro, cellular, in vivo, genetic, and pharmacological evidence to describe a specific neurochemical/molecular pathway involving $\mathrm{NE}$ metabolism and AEP that may explain the selective vulnerability of LC neurons to developing Tau pathology, the later demise of these cells, and the potential spread of pathology from the LC to the forebrain. Identification of the DOPEGAL-AEP-Tau N368 pathway lays the foundation for development of LC/AEP-based therapies that could slow the progression of $\mathrm{AD}$.

\section{Methods}

\section{Animals}

Tau P301S mice on a C57BL/6J background (line PS19), MAPT mice, 3XTg mice, and WT C57BL/6J mice were obtained from The Jackson
Laboratory (stock nos. 008169, 005491, 34830, and 000664, respectively). The $\mathrm{AEP}^{-/-}$mice were maintained on a mixed 129/Ola and C57BL/6 background. $\mathrm{DBH}^{-/-}$mice were maintained on a mixed 129/ SvEv and C57BL/6 background. Animal care and handling was performed according to the Declaration of Helsinki and Emory Medical School guidelines. Tau P301S mice were crossed with $\mathrm{DBH}^{-/-}$mice to generate Tau P301S/ $\mathrm{DBH}^{-/-}$mice. Adrenergic agonists and/or L-DOPS were used to rescue the $\mathrm{DBH}^{-/-}$embryonic lethality and male mating deficit, as described (49). MAPT mice were crossed with $\mathrm{AEP}^{-/-}$mice to generate $\mathrm{MAPT} / \mathrm{AEP}^{-/-}$mice. Investigators were blinded to the group allocation during the animal experiments.

\section{Human tissue samples}

Paraffin sections containing the LC from 5 postmortem AD cases of Braak stages IV-VI (age $74.5 \pm 11.2$ years, mean \pm SD) and 5 cognitively normal controls (age 73.9 \pm 12.7 years) were obtained from the Emory Alzheimer's Disease Research Center. AD was diagnosed according to the criteria of the Consortium to Establish a Registry for $\mathrm{AD}$ and the National Institute on Aging. Diagnoses were confirmed by the presence of amyloid plaques and NFTs in formalin-fixed tissue.

\section{Transfection and viral infection of cells}

Primary cortical and noradrenergic neurons were cultured as previously described (50). All rats were purchased from The Jackson Laboratory. HEK293 cells and SH-SY5Y cells were transfected with plasmids encoding Tau, SiDBH, MAO-A, or MAO-B using polyethylenimine or Lipofectamine 3000 (Thermo Fisher Scientific). AAV-Tau, AAV-Tau 


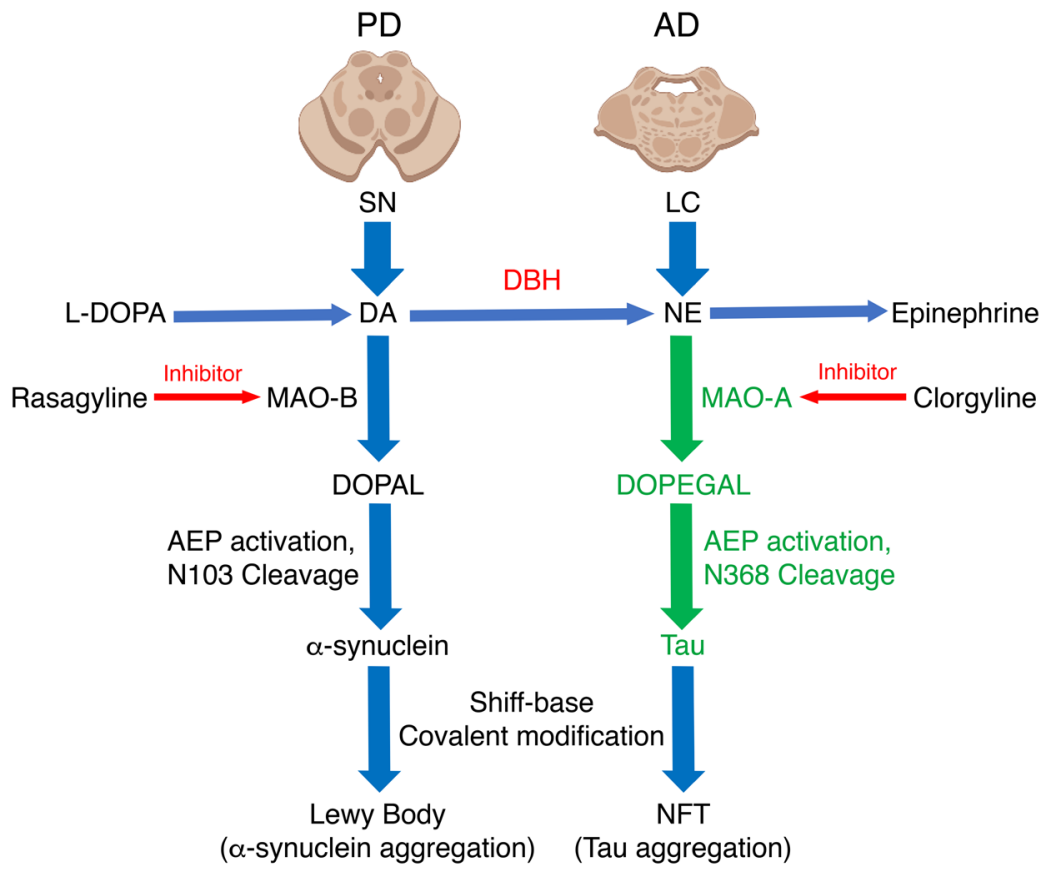

Figure 12. A model for the selective vulnerability by DA or NE metabolites, monoamine oxidase, and AEP in PD and AD. DOPAL, which is a DA metabolite by MAO-B, has a toxic effect in the substantia nigra in the progression of PD by mediating AEP activation and $\alpha$-synuclein toxicity. Similarly, MAO-A and its toxic metabolite of NE, DOPEGAL, induce Tau toxicity in noradrenergic cells of LC in the early stage of $A D$ progression.
P301S, AAV-Tau 1-368, and AAV Tau N225/368A were used for infection in primary neuron and SH-SY5Y (ATCC CRL-2266) neuroblastoma cells. Neurotoxicity was analyzed using LDH assay (CytoTox 96 Non-Radioactive Cytotoxicity Assay, Promega).

\section{Western blot analysis}

Cultured cells were lysed in lysis buffer (50 mM Tris, pH 7.4, $40 \mathrm{mM}$ $\mathrm{NaCl}, 1 \mathrm{mM}$ EDTA, 0.5\% Triton X-100, $1.5 \mathrm{mM} \mathrm{Na}_{3} \mathrm{VO}_{4}, 50 \mathrm{mM} \mathrm{NaF}$, $10 \mathrm{mM}$ sodium pyrophosphate, $10 \mathrm{mM}$ sodium $\beta$-glycerophosphate, supplemented with a cocktail of protease inhibitors) and centrifuged for 15 minutes at $16,000 \mathrm{~g}$. The supernatant was boiled in SDS loading buffer. After SDS-PAGE, the samples were transferred to a nitrocellulose membrane. Primary antibodies to the following targets were used: Tau5 (Santa Cruz Biotechnology, SC-58860), AT-8 (Thermo Fisher Scientific, MN1020), HT7 (Thermo Fisher Scientific, MN1000), $\beta$-actin (SigmaAldrich, A5316), MAO-B (GeneTex, GTX113771), MAO-A (Sigma-Aldrich, SAB1410774), TH (Abcam, AB112), AEP (Cell Signaling Technology, 93627S), Iba1 (VWR, 019-19741), and Tau N368 (custom antibody, 18).

\section{AEP activity assay}

Assay buffer (20 mM citric acid, $60 \mathrm{mM} \mathrm{Na} \mathrm{HPO}_{4}, 1 \mathrm{mM}$ EDTA, 0.1\% 3-[(3-cholamidopropyl)dimethylammonio]-1-propanesulfonate [CHAPS], and $1 \mathrm{mM}$ DTT, pH 6.0) of $100 \mu \mathrm{L}$ including $100 \mu \mathrm{M}$ AEP substrate Z-Ala-Ala-Asn-AMC (ABchem) was added to cell or tissue lysates. AMC released by substrate cleavage was quantified using a fluorescence plate reader at $460 \mathrm{~nm}$ for 1 hour in kinetic mode.

\section{MAO-A activity assay}

Cell or tissue lysates $(20 \mu \mathrm{g})$ were incubated with the working solution of $100 \mu \mathrm{L}$ containing $400 \mu \mathrm{M}$ AmplexÒ Red reagent, $2 \mathrm{U} / \mathrm{mL}$ HRP, and 2-mM p-tyramine substrate with MAO-B inhibitor, pargyline (Molecular Probes). The fluorescence of MAO-A activity was measured in a fluorescence plate reader using excitation in the range of $530-560 \mathrm{~nm}$ and emission at $590 \pm 10 \mathrm{~nm}$ at $37^{\circ} \mathrm{C}$ for 2 hours in kinetic mode.

\section{HPLC analysis}

LC tissue was resuspended in $150 \mu \mathrm{L}$ ice-cold $0.1 \mathrm{M}$ perchloric acid (PCA) containing $0.1 \mathrm{mM}$ EDTA and sonicated on ice by probe sonication on setting 3 with a $30 \%$ duty cycle. Homogenates were centrifuged at $10,000 \times g$ for 10 minutes at $4^{\circ} \mathrm{C}$, and supernatants were transferred into 0.22- $\mu \mathrm{M}$ PVDF microcentrifuge filter tubes to remove any remaining particulate matters at $5000 \times \operatorname{rpm}$ for 5 minutes at $4^{\circ} \mathrm{C}$. Monoamine concentrations were determined by reverse phase HPLC with electrochemical detection. Tissue protein pellets were dissolved in $500-\mu \mathrm{L}$ $2 \%$ SDS. Protein quantification was carried out in triplicate in 96-well microplates with SpectraMax M5e spectrophotometer (Molecular Devices) using the BCA method (Pierce BCA Protein Assay Kit, Thermo Fisher Scientific). For HPLC, an autosampler (ESA) 5600A CoulArray detection system, equipped with an ESA Model 584 pump and an ESA 542 refrigerated autosampler was used. Separations were performed at $23^{\circ} \mathrm{C}$ using an Xterra RP $4.6 \times 250 \mathrm{~mm}, 3.5-\mu \mathrm{m}$ particle size, C18 column (Waters). The mobile phase consisted of $72 \mathrm{mM}$ sodium hydrogen phosphate, $28 \mathrm{mM}$ citric acid, $0.6 \mathrm{mM}$ 1-octanesulfonic acid sodium, and 0.2 mM EDTA at pH 5.35. A 20- $\mu \mathrm{L}$ sample was injected. The samples were eluted isocratically at $0.5 \mathrm{ml} / \mathrm{min}$ and detected using a 6210 electrochemical cell. Analytical cell potentials were $-175,100$, and $350 \mathrm{mV}$. The analytes were identified by the matching criteria of retention time to known standards. Compounds were quantified by comparing peak areas with those of standards on the dominant sensor.

\section{ThioT assay}

ThioT stock solution $(3 \mathrm{mM})$ was filtered through a $0.2-\mu \mathrm{m}$ syringe filter (Sigma-Aldrich, catalog T3516). The stock solution was diluted into the aggregation buffer ( $20 \mathrm{mM}$ Tris, $\mathrm{pH} 7.4,100 \mathrm{mM} \mathrm{NaCl}, 1 \mathrm{mM}$ EDTA) to generate the working solution $(30 \mu \mathrm{M})$. ThioT working solution with heparin $(30 \mu \mathrm{M})$, recombinant Tau $(7 \mu \mathrm{M})$, and DOPEGAL $(500 \mu \mathrm{M})$ was incubated on an orbital shaker at $37^{\circ} \mathrm{C}$ and excited at $440 \mathrm{~nm}$ and emitted at $482 \mathrm{~nm}$ to measure the fluorescence intensity on the plater reader (BioTek, 251639). Fluorescence values (exci- 
tation at $440 \mathrm{~nm}$ and emission $482 \mathrm{~nm}$ ) onto the plate reader (BioTek, 251639) were recorded for 5 days.

\section{Immunostaining}

Paraffin-embedded human brain sections or free-floating mouse brain sections sliced by cryotome were treated with $0.3 \% \mathrm{H}_{2} \mathrm{O}_{2}$ for 10 minutes. Sections were washed 3 times in PBS and blocked in 1\% BSA, $0.3 \%$ Triton $\mathrm{X}-100$ for 30 minutes, followed by overnight incubation with TH antibody (1:1000), anti-Tau5, anti-Tau N368, or AT-8 antibody (1:500) at $4^{\circ} \mathrm{C}$. The signal was developed using a Histostain-SP kit (Invitrogen, Thermo Fisher Scientific). For IF, sections were incubated overnight with various primary antibodies at $4^{\circ} \mathrm{C}$. Then, the sections were incubated with the matched fluoroconjugated secondary antibody for 2 hours at room temperature, followed by 3 washes in PBS. The slides were washed 3 times in PBS and covered with a glass using mounting solution, after DAPI staining for 5 minutes.

\section{Stereotaxic injection}

AAV-PRSx8-mCherry, AAV-PRSx8-Tau, AAV-Tau P301S, AAV-Tau N255/368A, or AAV-MAO-A were injected into the LC region of mice. Three-month-old mice of each group were anesthetized with isoflurane (Piramal Healthcare). Meloxicam $(2 \mathrm{mg} / \mathrm{kg}$ ) was injected s.c. for analgesia (Loxicom, Norbrook). Unilateral intracerebral injection of virus was performed stereotaxically at the following coordinates: anteroposterior $-5.4 \mathrm{~mm}$ and mediolateral $-1.2 \mathrm{~mm}$ relative to breg$\mathrm{ma}$, and dorsoventral $-3.7 \mathrm{~mm}$ from the dural surface. Two microliters of viral suspension was injected into each site using a 10- $\mu \mathrm{L}$ Hamilton syringe with a fixed needle at a rate of $0.25 \mu \mathrm{L} / \mathrm{min}$. The needle remained in place for 5 minutes after the viral suspension was completely injected and then was slowly removed over 2 minutes. The mice were placed on a heating pad until recovery from the anesthesia.

\section{Behavioral testing}

$M W M$. Mice were trained in a round, water-filled tub (52-in. diameter) in an environment with extra maze cues. Each subject was given 4 trials/day for 5 consecutive days, with a 15 -minute intertrial interval. The maximum trial length was 60 seconds, and if subjects did not reach the platform in the allotted time frame, they were manually guided to it. Following the 5 days of task acquisition, a probe trial was presented, during which time the platform was removed and the percentage of time spent in the quadrant that previously contained the escape platform during task acquisition was measured over 60 seconds. All trials were analyzed for latency and swim speed by MazeScan (CleverSys Inc.). We monitored body weight, visual ability, and swim speed during behavioral test to verify that the deficits did not result from motor dysfunction.

Fear conditioning. The ability to form and retain an association between an aversive experience and environmental cues was tested with a standard fear-conditioning paradigm occurring over a period of 3 days. Mice were placed in the fear-conditioning apparatus $\left(7^{\prime \prime} \mathrm{W}, 7^{\prime \prime}\right.$ $\mathrm{D} \times 12^{\prime \prime} \mathrm{H}$, Coulbourn Instruments) composed of Plexiglas with a metal shock grid floor and allowed to explore the enclosure for 3 minutes. Following this habituation period, 3 conditioned stimulus (CS)-unconditioned stimulus (US) pairings were presented with a 1-minute intertrial interval. The CS consisted of a 20 -second $85-\mathrm{db}$ tone, and the US consisted of 2 seconds of a $0.5-\mathrm{mA}$ footshock, which was coterminated with each CS presentation. One minute following the last CS-US presentation, mice were returned to their home cage. On day 2 , the mice were presented with a context test, during which subjects were placed in the same chamber used during conditioning on day 1 , and the amount of freezing was recorded via camera (software provided by Colbourn). No shocks were given during the context test. On day 3, a tone test was presented, during which time subjects were exposed to the CS in a novel compartment. Initially, animals were allowed to explore the novel context for 2 minutes. Then the $85-\mathrm{db}$ tone was presented for 6 minutes, and the amount of freezing behavior was recorded.

\section{Quantification and statistical analysis}

All cell culture data are expressed as mean \pm SEM from 3 or more independent experiments, and the level of significance between 2 groups was assessed with Student's $t$ test. For experiments consisting of more than 2 groups, 1-way or 2-way ANOVA followed by Tukey's post hoc test was applied. A $P$ value of less than 0.05 was considered statistically significant. Sample sizes were determined by Power and Precision (Biostat).

\section{Study approval}

The protocol for animal experiments was reviewed and approved by the Emory Institutional Animal Care and Use Committee. The study using human tissue samples was approved by the Biospecimen Committee, and informed consent was obtained from the subjects prior to their deaths.

\section{Author contributions}

$\mathrm{KY}$ conceived the project, designed the experiments, analyzed the data, and wrote the manuscript. SSK designed and performed most of the experiments and analyzed the data. XL prepared primary neurons and assessed animal experiments. CJ performed the crosses, pharmacological embryonic lethality rescue, and genotyping for experiments with the $\mathrm{DBH}^{-/}$mice. EHA and JX performed Tau purification and some of the in vitro experiments. FPM prepared the uncleavable Tau virus. HRL and XY assisted with data analysis and interpretation and critically read the manuscript. DW helped design the experiments and edit the manuscript.

\section{Acknowledgments}

This work was supported by the NIH (R01AG051538; RF1 AG061175 to KY and RF1AG047667 to DW). We thank the Emory Alzheimer's Disease Research Center for postmortem human AD and healthy control samples. We thank the University of Washington Alzheimer's Disease Research Center (NIH P50AG005136) and the Kaiser Permanente Adult Changes in Thought Study (NIH U01 AG006781) research subjects and their families for brain donation, a tremendous gift to science. We thank Allison Beller for coordination of tissue requests, subject selection, and tissue transfer, Kim Howard for histological support, and Cheryl Strauss for helpful editing of the manuscript. This study was supported in part by the Rodent Behavioral Core (RBC) and Viral Vector Core, which are subsidized by the Emory University School of Medicine and are part of the Emory Integrated Core Facilities. Additional support was provided by the Emory Neuroscience NINDS Core Facilities (P30NS055077). Further support was provided by the Georgia Clinical \& Translational Science Alliance of the NIH under Award Number UL1TR002378.

Address correspondence to: Keqiang Ye, 615 Michael Street, Whitehead Bldg, Room \#141, Atlanta, Georgia 30322, USA. Phone: 404.712.2814; Email: kye@emory.edu. 
1. Canter RG, Penney J, Tsai LH. The road to restoring neural circuits for the treatment of Alzheimer's disease. Nature. 2016;539(7628):187-196.

2. Foote SL, Bloom FE, Aston-Jones G. Nucleus locus ceruleus: new evidence of anatomical and physiological specificity. Physiol Rev. 1983;63(3):844-914.

3. Braak H, Thal DR, Ghebremedhin E, Del Tredici K. Stages of the pathologic process in Alzheimer disease: age categories from 1 to 100 years. J Neuropathol Exp Neurol. 2011;70(11):960-969.

4. Braak H, Del Tredici K. Where, when, and in what form does sporadic Alzheimer's disease begin? Curr Opin Neurol. 2012;25(6):708-714

5. Ehrenberg AJ, et al. Quantifying the accretion of hyperphosphorylated tau in the locus coeruleus and dorsal raphe nucleus: the pathological building blocks of early Alzheimer's disease. Neuropathol Appl Neurobiol. 2017;43(5):393-408.

6. Theofilas P, et al. Locus coeruleus volume and cell population changes during Alzheimer's disease progression: a stereological study in human postmortem brains with potential implication for early-stage biomarker discovery. Alzheimers Dement. 2017;13(3):236-246.

7. Weinshenker D. Long road to ruin: noradrenergic dysfunction in neurodegenerative disease. Trends Neurosci. 2018;41(4):211-223.

8. Wilson RS, et al. Brainstem aminergic nuclei and late-life depressive symptoms. JAMA Psychiatry. 2013;70(12):1320-1328.

9. Heneka MT, et al. Locus ceruleus degeneration promotes Alzheimer pathogenesis in amyloid precursor protein 23 transgenic mice. J Neurosci. 2006;26(5):1343-1354.

10. Hammerschmidt T, et al. Selective loss of noradrenaline exacerbates early cognitive dysfunction and synaptic deficits in APP/PS1 mice. Biol Psychiatry. 2013;73(5):454-463.

11. Chalermpalanupap T, et al. Locus coeruleus ablation exacerbates cognitive deficits, neuropathology, and lethality in P301S Tau transgenic mice. J Neurosci. 2018;38(1):74-92.

12. Kelly SC, He B, Perez SE, Ginsberg SD, Mufson EJ, Counts SE. Locus coeruleus cellular and molecular pathology during the progression of Alzheimer's disease. Acta Neuropathol Commun. 2017;5(1):8

13. Heneka MT, et al. Locus ceruleus controls Alzheimer's disease pathology by modulating microglial functions through norepinephrine. Proc Natl Acad Sci U S A. 2010;107(13):6058-6063.

14. Rorabaugh JM, et al. Chemogenetic locus coeruleus activation restores reversal learning in a rat model of Alzheimer's disease. Brain. 2017;140(11):3023-3038.

15. Jucker M, Walker LC. Pathogenic protein seeding in Alzheimer disease and other neurodegenerative disorders. Ann Neurol. 2011;70(4):532-540.

16. Chalermpalanupap T, Weinshenker D, Rorabaugh JM. Down but not out: the consequences of pretangle Tau in the locus coeruleus. Neural Plast. 2017;2017:7829507.

17. Zhang Z, Xie M, Ye K. Asparagine endopeptidase is an innovative therapeutic target for neurodegenerative diseases. Expert Opin Ther Targets. 2016;20(10):1237-1245.

18. Zhang Z, et al. Cleavage of tau by asparagine endopeptidase mediates the neurofibrillary pathology in Alzheimer's disease. Nat Med. 2014;20(11):1254-1262.

19. Zhang Z, et al. Delta-secretase cleaves amyloid precursor protein and regulates the pathogenesis in Alzheimer's disease. Nat Commun. 2015;6:8762.

20. Zhang Z, et al. Inhibition of delta-secretase improves cognitive functions in mouse models of Alzheimer's disease. Nat Commun. 2017;8:14740.

21. Kang SS, et al. $\alpha$-Synuclein stimulation of monoamine oxidase-B and legumain protease mediates the pathology of Parkinson's disease. ЕМВО J. 2018;37(12):e98878.

22. Zhang Z, et al. Asparagine endopeptidase cleaves $\alpha$-synuclein and mediates pathologic activities in Parkinson's disease. Nat Struct Mol Biol. 2017;24(8):632-642.

23. Burke WJ, Schmitt CA, Gillespie KN, Li SW. Norepinephrine transmitter metabolite is a selective cell death messenger in differentiated rat pheochromocytoma cells. Brain Res. 1996;722(1-2):232-235.

24. Burke WJ, Li SW, Schmitt CA, Xia P, Chung HD, Gillespie KN. Accumulation of 3,4-dihydroxyphenylglycolaldehyde, the neurotoxic monoamine oxidase A metabolite of norepinephrine, in locus ceruleus cell bodies in Alzheimer's disease: mechanism of neuron death. Brain Res. 1999;816(2):633-637.

25. Burke WJ, et al. Neurotoxicity of MAO metabolites of catecholamine neurotransmitters: role in neurodegenerative diseases. Neurotoxicology. 2004;25(1-2):101-115

26. Burke WJ, et al. Catecholamine monoamine oxidase a metabolite in adrenergic neurons is cytotoxic in vivo. Brain Res. 2001;891(1-2):218-227.

27. Burke WJ, Kristal BS, Yu BP, Li SW, Lin TS. Norepinephrine transmitter metabolite generates free radicals and activates mitochondrial permeability transition: a mechanism for DOPEGAL-induced apoptosis. Brain Res. 1998;787(2):328-332.

28. Burke WJ, Mattammal MB, Marshall GL, Chung $\mathrm{H}$. Detection of 3,4-dihydroxyphenylglycolaldehyde in human brain by high-performance liquid chromatography. Anal Biochem. 1989;180(1):79-84

29. Cohen G. Monoamine oxidase, hydrogen peroxide, and Parkinson's disease. Adv Neurol. 1987;45:119-125.

30. Biedler JL, Roffler-Tarlov S, Schachner M, Freedman LS. Multiple neurotransmitter synthesis by human neuroblastoma cell lines and clones. Cancer Res. 1978;38(11 pt 1):3751-3757.

31. Levin EY, Levenberg B, Kaufman S. The enzymatic conversion of 3,4-dihydroxyphenylethylamine to norepinephrine. J Biol Chem. 1960;235:2080-2086.

32. May JM, Qu ZC, Meredith ME. Mechanisms of ascorbic acid stimulation of norepinephrine synthesis in neuronal cells. Biochem Biophys Res Commun. 2012;426(1):148-152.

33. Bourdélat-Parks BN, et al. Effects of dopamine beta-hydroxylase genotype and disulfiram inhibition on catecholamine homeostasis in mice. Psychopharmacology (Berl). 2005;183(1):72-80.

34. Vazey EM, Aston-Jones G. Designer receptor manipulations reveal a role of the locus coe- ruleus noradrenergic system in isoflurane general anesthesia. Proc Natl Acad Sci U S A. 2014;111(10):3859-3864.

35. Burke WJ, et al. Aggregation of $\alpha$-synuclein by DOPAL, the monoamine oxidase metabolite of dopamine. Acta Neuropathol. 2008;115(2):193-203.

36. Follmer C, et al. Oligomerization and membrane-binding properties of covalent adducts formed by the interaction of $\alpha$-synuclein with the toxic dopamine metabolite 3,4-dihydroxyphenylacetaldehyde (DOPAL). J Biol Chem. 2015;290(46):27660-27679.

37. Werner-Allen JW, Monti S, DuMond JF, Levine $\mathrm{RL}, \mathrm{Bax} \mathrm{A}$. Isoindole linkages provide a pathway for DOPAL-mediated cross-linking of $\alpha$-synuclein. Biochemistry. 2018;57(9):1462-1474.

38. Goldstein DS, et al. Vesicular uptake blockade generates the toxic dopamine metabolite 3,4-dihydroxyphenylacetaldehyde in PC12 cells: relevance to the pathogenesis of Parkinson's disease. J Neurochem. 2012;123(6):932-943.

39. Burke WJ, Li SW, Williams EA, Nonneman R, Zahm DS. 3,4-Dihydroxyphenylacetaldehyde is the toxic dopamine metabolite in vivo: implications for Parkinson's disease pathogenesis. Brain Res. 2003;989(2):205-213.

40. Wang $\mathrm{ZH}$, et al. C/EBP $\beta$ regulates delta-secretase expression and mediates pathogenesis in mouse models of Alzheimer's disease. Nat Commun. 2018;9(1):1784

41. Shih JC, Chen K, Ridd MJ. Role of MAO A and B in neurotransmitter metabolism and behavior. Pol JPharmacol. 1999;51(1):25-29.

42. Shih JC, Chen K, Ridd MJ. Monoamine oxidase: from genes to behavior. Annu Rev Neurosci. 1999;22:197-217.

43. Adolfsson R, Gottfries CG, Oreland L, Wiberg A, Winblad B. Increased activity of brain and platelet monoamine oxidase in dementia of Alzheimer type. Life Sci. 1980;27(12):1029-1034.

44. Schedin-Weiss S, et al. Monoamine oxidase B is elevated in Alzheimer disease neurons, is associated with $\gamma$-secretase and regulates neuronal amyloid beta-peptide levels. Alzheimers Res Ther. 2017;9(1):57.

45. Kang SS, et al. TrkB neurotrophic activities are blocked by $\alpha$-synuclein, triggering dopaminergic cell death in Parkinson's disease. Proc Natl Acad Sci U S A. 2017;114(40):10773-10778.

46. Bondareff W, Mountjoy CQ, Roth M. Loss of neurons of origin of the adrenergic projection to cerebral cortex (nucleus locus ceruleus) in senile dementia. Neurology. 1982;32(2):164-168.

47. Oddo S, et al. Triple-transgenic model of Alzheimer's disease with plaques and tangles: intracellular A $\beta$ and synaptic dysfunction. Neuron. 2003;39(3):409-421.

48. Andorfer C, et al. Hyperphosphorylation and aggregation of tau in mice expressing normal human tau isoforms. J Neurochem. 2003;86(3):582-590.

49. Thomas SA, Marck BT, Palmiter RD, Matsumoto $A M$. Restoration of norepinephrine and reversal of phenotypes in mice lacking dopamine betahydroxylase. J Neurochem. 1998;70(6):2468-2476.

50. Liu X, Ye K, Weinshenker D. Norepinephrine protects against amyloid- $\beta$ toxicity via TrkB. JAlzheimers Dis. 2015;44(1):251-260. 